\title{
REAL EQUIVALENCE OF COMPLEX MATRIX PENCILS AND COMPLEX PROJECTIONS OF REAL SEGRE VARIETIES*
}

\author{
ADAM COFFMAN ${ }^{\dagger}$
}

\begin{abstract}
Quadratically parametrized maps from a product of real projective spaces to a complex projective space are constructed as the composition of the Segre embedding with a projection. A classification theorem relates equivalence classes of projections to equivalence classes of complex matrix pencils. One low-dimensional case is a family of maps whose images are ruled surfaces in the complex projective plane, some of which exhibit hyperbolic CR singularities. Another case is a set of maps whose images in complex projective 4-space are projections of the real Segre threefold.
\end{abstract}

Key words. Matrix pencil, Matrix equivalence, Ruled surface, Segre embedding, CR singularity.

AMS subject classifications. 15A22, 14E05, 14J26, 14P05, 32V40, 51N15.

1. Introduction. We consider maps into a complex projective space, of the form

$$
\left(\left[u_{0}: u_{1}: \cdots: u_{\ell}\right],\left[v_{0}: v_{1}: \cdots: v_{m}\right]\right) \mapsto\left[P_{0}: P_{1}: \cdots: P_{n}\right]
$$

where the $u_{j}$ and $v_{j}$ are real homogeneous coordinates, and each $P_{k}$ is a bihomogeneous quadratic polynomial with complex coefficients, linear in $u_{j}$ and $v_{j}$ separately. Outside the common zero locus of $P_{0}, \ldots, P_{n}$, such a parametrization defines a smooth map $\mathbb{R} P^{\ell} \times \mathbb{R} P^{m} \rightarrow \mathbb{C} P^{n}$. A natural classification of such maps is to say that two are equivalent if they are related by real linear coordinate changes in the domain and a complex linear transformation of the target. After a brief review of the necessary fundamentals in Section 2, the practical approach to the equivalence of these quadratic parametrizations will be its relationship to the equivalence of matrix pencils; see Proposition 2.11. Section 3 gives an overview of the geometric features of quadratically parametrized subsets of $\mathbb{C} P^{n}$, which can include singularities, self-intersections, complex tangents, and more subtle projective-geometric properties. Some low-dimensional cases will be investigated in detail, namely, maps $\mathbb{R} P^{1} \times \mathbb{R} P^{1} \rightarrow \mathbb{C} P^{2}$ in Section 4 , and $\mathbb{R} P^{2} \times \mathbb{R} P^{1} \rightarrow \mathbb{C} P^{4}$ in Section 5 .

The constructions of this article are related to those of $[5,6]$, where other kinds of quadratically parametrized real subvarieties of complex projective space are classi-

\footnotetext{
${ }^{*}$ Received by the editors March 4, 2008. Accepted for publication November 27, 2008. Handling Editor: Raphael Loewy.

${ }^{\dagger}$ Department of Mathematical Sciences, Indiana University - Purdue University Fort Wayne, 2101 E. Coliseum Blvd., Fort Wayne, Indiana 46805-1499, USA (CoffmanA@ipfw.edu).
} 
fied algebraically and analyzed geometrically. The geometric motivation for the program of studying such real rational maps is to find interesting, naturally occurring, concretely expressible examples of real subvarieties of high codimension in complex manifolds, which are either totally real, or which have CR singularities.

\section{The projective geometric construction.}

2.1. General background. Most of the maps and objects to be used are standard in elementary projective geometry and have appeared in [5] or [6], and we briefly recall them here to fix notation. To start, let $\mathbb{K}$ be a field, which later will be either $\mathbb{R}$ or $\mathbb{C}$, and let $\mathbb{K} P^{n}$ be the set of one-dimensional subspaces of $\mathbb{K}^{n+1}$, so that a non-zero column vector $\mathbf{z}=\left(z_{0}, \ldots, z_{n}\right)^{T} \in \mathbb{K}^{n+1}$ spans a line $z=\left[z_{0}: \cdots: z_{n}\right] \in \mathbb{K} P^{n}$.

For whole numbers $\ell, m$, the following map is formed by all the $(\ell+1)(m+1)$ quadratic monomials $z_{i} w_{j}$ in the components of two vectors $\mathbf{z}$ and $\mathbf{w}$ :

$$
\begin{aligned}
\mathbf{s}_{\mathbb{K}}^{\ell m}: \mathbb{K}^{\ell+1} \times \mathbb{K}^{m+1} & \rightarrow \mathbb{K}^{(\ell+1)(m+1)}: \\
(\mathbf{z}, \mathbf{w}) & \mapsto\left(z_{0} w_{0}, z_{0} w_{1}, \ldots, z_{0} w_{m}, \ldots, z_{\ell} w_{0}, \ldots, z_{\ell} w_{m}\right)^{T}
\end{aligned}
$$

Since it has the property that $\mathbf{s}_{\mathbb{K}}^{\ell m}(\lambda \mathbf{z}, \mu \mathbf{w})=\lambda \mu \mathbf{s}_{\mathbb{K}}^{\ell m}(\mathbf{z}, \mathbf{w}) \neq \mathbf{0}$ for all $\lambda, \mu \in \mathbb{K} \backslash\{0\}$, $(\mathbf{z}, \mathbf{w}) \in\left(\mathbb{K}^{\ell+1} \backslash\{\mathbf{0}\}\right) \times\left(\mathbb{K}^{m+1} \backslash\{\mathbf{0}\}\right)$, it induces a map:

$$
\begin{aligned}
s_{\mathbb{K}}^{\ell m}: \mathbb{K} P^{\ell} \times \mathbb{K} P^{m} & \rightarrow \mathbb{K} P^{\ell m+\ell+m}: \\
(z, w) & \mapsto\left[z_{0} w_{0}: z_{0} w_{1}: \cdots: z_{0} w_{m}: \cdots: z_{\ell} w_{0}: \cdots: z_{\ell} w_{m}\right],
\end{aligned}
$$

called the Segre map. Define a vector space isomorphism from the space of $c \times d$ matrices to the space of column $(c d)$-vectors by stacking the columns of the matrix

$$
\text { vec }: M(c \times d, \mathbb{K}) \rightarrow \mathbb{K}^{c d}:\left[\mathbf{z}^{1} \cdots \mathbf{z}^{d}\right]_{c \times d} \mapsto\left[\begin{array}{c}
\mathbf{z}^{1} \\
\vdots \\
\mathbf{z}^{d}
\end{array}\right]_{(c d) \times 1}
$$

This is the well-known vectorization map from matrix algebra [14]. Denote its inverse by $\mathbf{k}: \mathbb{K}^{c d} \rightarrow M(c \times d, \mathbb{K})$.

The composition of the map $\mathbf{s}_{\mathbb{K}}^{\ell m}$ with the isomorphism $\mathbf{k}$ (in the case $c=m+1$, $d=\ell+1$ ) has the following interpretation in terms of matrix multiplication:

$$
\left(\mathbf{k} \circ \mathbf{s}_{\mathbb{K}}^{\ell m}\right)(\mathbf{z}, \mathbf{w})=\mathbf{w} \mathbf{z}^{T} .
$$

The transpose $\mathbf{z}^{T}$ is a row vector; the RHS is a $(m+1) \times(\ell+1)$ matrix of rank $\leq 1$. 
2.2. Complex projective geometry. We continue here with some elementary constructions, as in the previous subsection, but with $\mathbb{K}=\mathbb{C}$, so we are in the familiar territory of complex projective geometry. We also will consider projective spaces with their usual topological and analytic structure; for example, the Segre map $s_{\mathbb{C}}^{\ell m}$ : $\mathbb{C} P^{\ell} \times \mathbb{C} P^{m} \rightarrow \mathbb{C} P^{\ell m+\ell+m}$ is a holomorphic embedding of complex manifolds [12]. It will sometimes be convenient to abbreviate $\mathbf{s}_{\mathbb{C}}^{\ell m}=\mathbf{s}$ and $s_{\mathbb{C}}^{\ell m}=s$.

The next ingredients in the construction are an integer $n$ such that $0 \leq n \leq$ $\ell m+\ell+m$, and a $(n+1) \times(\ell+1)(m+1)$ matrix $\mathbf{P}$ with complex entries and full rank $n+1 \leq(\ell+1)(m+1)$, called the coefficient matrix. The linear transformation $\mathbb{C}^{(\ell+1)(m+1)} \rightarrow \mathbb{C}^{n+1}$ (also denoted by $\mathbf{P}$ ) induces a projection map $P: \mathbb{C} P^{\ell m+\ell+m} \rightarrow$ $\mathbb{C} P^{n}$, which is well-defined for all elements in the domain except those lines in the kernel of $\mathbf{P}$.

So, the composition $P \circ s$ is a well-defined map $\mathbb{C} P^{\ell} \times \mathbb{C} P^{m} \rightarrow \mathbb{C} P^{n}$ if the image of $\mathbf{s}_{\mathbb{C}}^{\ell m}$ contains no lines in the kernel of $\mathbf{P}$. When the $(n+1)(\ell+1)(m+1)$ entries of the matrix $\mathbf{P}$ are used as complex coefficients $p_{k}^{i, j}$ of quadratic polynomials

$$
P_{k}=\sum_{j=0}^{m} \sum_{i=0}^{\ell} p_{k}^{i, j} z_{i} w_{j}
$$

the map $P \circ s$ is of the form

$$
\left(\left[z_{0}: \cdots: z_{\ell}\right],\left[w_{0}: \cdots: w_{m}\right]\right) \mapsto\left[P_{0}: \cdots: P_{n}\right] .
$$

It will be convenient to follow some of the recent literature on implicitization (e.g., [1, 3, 9]), and borrow some terminology from the classical (and not unrelated; see $[15, \S I I I .1])$ theory of linear systems of quadrics.

Definition 2.1. Given a coefficient matrix $\mathbf{P}$, if $\mathbf{z}$ and $\mathbf{w}$ are non-zero vectors such that $(\mathbf{P} \circ \mathbf{s})((\mathbf{z}, \mathbf{w}))=\mathbf{0} \in \mathbb{C}^{n+1}$, then the point $(z, w) \in \mathbb{C} P^{\ell} \times \mathbb{C} P^{m}$ will be called a base point of the composite map $P \circ s$.

We will continue to call $\mathbb{C} P^{\ell} \times \mathbb{C} P^{m}$ the domain of the parametric map $P \circ s$, but with the understanding that if $(z, w)$ is a base point, then $(P \circ s)((z, w))$ is undefined, in the sense that $(z, w)$ has no image in the target $\mathbb{C} P^{n}$. We will also continue to call $(P \circ s)\left(\mathbb{C} P^{\ell} \times \mathbb{C} P^{m}\right)=\left\{(P \circ s)((z, w)) \in \mathbb{C} P^{n}:(z, w)\right.$ is not a base point $\}$ the image of $P \circ s$, even though it may not be a closed subset of the target if there are base points.

EXAMPLE 2.2. The $\ell=m=1, n=2$ case is in the assumed dimension range. A $3 \times 4$ matrix $\mathbf{P}$ with rank 3 has a kernel equal to a line in $\mathbb{C}^{4}$, or a single point $x \in \mathbb{C} P^{3}$. $P \circ s: \mathbb{C} P^{1} \times \mathbb{C} P^{1} \rightarrow \mathbb{C} P^{2}$ is well-defined at every point in the domain if the point $x$ is not an element of the image of $s\left(\left(\left[z_{0}: z_{1}\right],\left[w_{0}: w_{1}\right]\right)\right)=\left[z_{0} w_{0}: z_{0} w_{1}: z_{1} w_{0}: z_{1} w_{1}\right]$, 
a complex surface in $\mathbb{C} P^{3}$. Otherwise, there is exactly one base point, and $P \circ s$ is defined on the complement of that point in $\mathbb{C} P^{1} \times \mathbb{C} P^{1}$.

The following proposition for maps without base points is an analogue of Theorem 2.2 of [6], and the proof given there, which uses a little complex analysis, can be easily adapted.

Proposition 2.3. Suppose $\mathbf{P}$ and $\mathbf{Q}$ are coefficient matrices so that the induced maps $P \circ s$ and $Q \circ s$ are equal, and well-defined at every point of $\mathbb{C} P^{\ell} \times \mathbb{C} P^{m}$. Then, there exists a non-zero constant $\nu \in \mathbb{C}$ so that $\mathbf{P}=\nu \mathbf{Q}$.

ExAmple 2.4. In general, to establish that $\mathbf{P}=\nu \mathbf{Q}$, it is not enough to check that $P \circ s=Q \circ s$ only on some open set. For example, with $\ell=m=n=1$, the coefficient matrix

$$
\mathbf{P}=\left[\begin{array}{llll}
1 & 0 & i & 0 \\
0 & 1 & 0 & i
\end{array}\right]
$$

defines a composite map $P \circ s:\left(\left[z_{0}: z_{1}\right],\left[w_{0}: w_{1}\right]\right) \mapsto\left[\left(z_{0}+i z_{1}\right) w_{0}:\left(z_{0}+i z_{1}\right) w_{1}\right]$, which is not defined on the line $\left\{\left([1: i],\left[w_{0}: w_{1}\right]\right)\right\}$ (the base point locus). For

$$
\mathbf{Q}=\left[\begin{array}{cccc}
1 & 0 & -i & 0 \\
0 & 1 & 0 & -i
\end{array}\right]
$$

the composite map is $Q \circ s:\left(\left[z_{0}: z_{1}\right],\left[w_{0}: w_{1}\right]\right) \mapsto\left[\left(z_{0}-i z_{1}\right) w_{0}:\left(z_{0}-i z_{1}\right) w_{1}\right]$. Its base point locus is the line $\left\{\left([1:-i],\left[w_{0}: w_{1}\right]\right)\right\}$, but $(Q \circ v)((z, w))=(P \circ v)((z, w))$ for every $(z, w) \in \mathbb{C} P^{1} \times \mathbb{C} P^{1}$ where both maps are defined.

Definition 2.5. For fixed whole numbers $\ell, m, n$, two coefficient matrices $\mathbf{P}$ and $\mathbf{Q}$ are c-equivalent if there exist three invertible matrices, $\mathbf{A}_{1} \in G L(\ell+1, \mathbb{C}), \mathbf{A}_{2} \in$ $G L(m+1, \mathbb{C}), \mathbf{B} \in G L(n+1, \mathbb{C})$ such that for all $(\mathbf{z}, \mathbf{w}) \in\left(\mathbb{C}^{\ell+1} \backslash\{\mathbf{0}\}\right) \times\left(\mathbb{C}^{m+1} \backslash\{\mathbf{0}\}\right)$,

$$
\mathbf{Q}(\mathbf{s}((\mathbf{z}, \mathbf{w})))=\mathbf{B} \mathbf{P}\left(\mathbf{s}\left(\left(\mathbf{A}_{1} \mathbf{z}, \mathbf{A}_{2} \mathbf{w}\right)\right)\right) \in \mathbb{C}^{n+1} .
$$

The following proposition is an analogue of Corollary 2.7 of [6], and a similar proof can be given, using Proposition 2.3 above.

Proposition 2.6. Given matrices $\mathbf{P}$ and $\mathbf{Q}$, let $P$ and $Q$ be the induced projections. If $\mathbf{P}$ and $\mathbf{Q}$ are c-equivalent, then there exist automorphisms $A_{1} \in P G L(\ell+$ $1, \mathbb{C}), A_{2} \in P G L(m+1, \mathbb{C}), B \in P G L(n+1, \mathbb{C})$ such that

$$
(Q \circ s)((z, w))=B\left((P \circ s)\left(\left(A_{1} z, A_{2} w\right)\right)\right)
$$

for all $(z, w) \in \mathbb{C} P^{\ell} \times \mathbb{C} P^{m}$ where both sides are defined. Conversely, if there exist $A_{1}, A_{2}$, and $B$ such that $Q$ and $P$ satisfy the above equation at every point $(z, w) \in$ $\mathbb{C} P^{\ell} \times \mathbb{C} P^{m}$, then $\mathbf{P}$ and $\mathbf{Q}$ are c-equivalent. 
The geometric idea is that the compositions $Q \circ s$ and $P \circ s$ are related by a re-parametrization $\left(A_{1}, A_{2}\right)$ of the domain, $\mathbb{C} P^{\ell} \times \mathbb{C} P^{m}$, and a coordinate change $B$ of the target, $\mathbb{C} P^{n}$. The converse part of the above proposition does not apply when either $P \circ s$ or $Q \circ s$ has a base point. Whether or not there are base points, the composite map $P \circ s$ may not be one-to-one, and may also have singular points, where its (complex) Jacobian has rank less than $\ell+m$.

The next proposition, relating c-equivalence to equivalence of matrix pencils, was stated and proved in [5] for the case $\ell=m$, and that proof can be easily adapted for the more general case.

Proposition 2.7. $\mathbf{P}$ and $\mathbf{Q}$ are c-equivalent if and only if there exist $\mathbf{A}_{1} \in$ $G L(\ell+1, \mathbb{C}), \mathbf{A}_{2} \in G L(m+1, \mathbb{C})$ such that the following $(\ell m+\ell+m-n)$-dimensional subspaces of $M((m+1) \times(\ell+1), \mathbb{C})$ are equal:

$$
\mathbf{k}(\operatorname{ker}(\mathbf{P}))=\mathbf{A}_{2}(\mathbf{k}(\operatorname{ker}(\mathbf{Q}))) \mathbf{A}_{1}^{T}
$$

Recall that rectangular matrices $\mathbf{R}, \mathbf{S} \in M(c \times d, \mathbb{C})$ are said to be equivalent [13] if there exist invertible complex matrices $\mathbf{A}_{1}, \mathbf{A}_{2}$ such that $\mathbf{R}=\mathbf{A}_{2} \mathbf{S} \mathbf{A}_{1}^{T}$. For each $D$, there is an obviously analogous relation on the set of $D$-dimensional complex linear subspaces of $M(c \times d, \mathbb{C})$ : given subspaces $U, V$ of $M(c \times d, \mathbb{C})$, if there exist invertible complex matrices $\mathbf{A}_{1}, \mathbf{A}_{2}$ such that for any $\mathbf{S} \in U, \mathbf{A}_{2} \mathbf{S} \mathbf{A}_{1}^{T} \in V$, then $U$ and $V$ will also be called equivalent.

In the case $\ell=m=1, n=2$, the c-equivalence classes of $3 \times 4$ coefficient matrices can be found by noticing that there are only two equivalence classes of onedimensional subspaces of $M(2 \times 2, \mathbb{C})$ : the class of pencils spanned by a rank 1 matrix, and the class of pencils spanned by a rank 2 matrix. This easy calculation appears in [5].

In terms of the geometry of the map $P \circ s$, the two c-equivalence classes correspond to the two well-known ways [12] to project the complex Segre surface in $\mathbb{C} P^{3}$ to a complex projective plane: the center of projection can be either on the surface or off the surface, as mentioned in Example 2.2.

EXAMPLE 2.8. In the rank 1 case, the map $P \circ s: \mathbb{C} P^{1} \times \mathbb{C} P^{1} \rightarrow \mathbb{C} P^{2}$ has exactly one base point; since $\mathbf{k}(\operatorname{ker}(\mathbf{P}))$ is in the image of $\mathbf{s}$, it is equivalent to

$$
\left\{\lambda\left[\begin{array}{ll}
1 & 0 \\
0 & 0
\end{array}\right]: \lambda \in \mathbb{C}\right\}
$$


By Proposition 2.7, $\mathbf{P}$ is c-equivalent to

$$
\mathbf{Q}=\left[\begin{array}{llll}
0 & 1 & 0 & 0 \\
0 & 0 & 1 & 0 \\
0 & 0 & 0 & 1
\end{array}\right]
$$

The composite map, $Q \circ s:\left(\left[z_{0}: z_{1}\right],\left[w_{0}: w_{1}\right]\right) \mapsto\left[z_{0} w_{1}: z_{1} w_{0}: z_{1} w_{1}\right]$, has its base point at $([1: 0],[1: 0])$. Its restriction to an affine neighborhood is one-to-one and nonsingular: $([z: 1],[w: 1]) \mapsto[z: w: 1]$, but one of the two lines outside this neighborhood is mapped to a point: $([1: 0],[w: 1]) \mapsto[1: 0: 0]$; similarly, the other line is mapped to another point: $([z: 1],[1: 0]) \mapsto[0: 1: 0]$. The singular locus can be described with a bihomogeneous expression:

$$
\Sigma_{1}=\left\{z_{1} w_{1}=0\right\} \backslash\{([1: 0],[1: 0])\} .
$$

The image of $Q \circ s$ in $\mathbb{C} P^{2}$ is the set $\left\{\left[Z_{0}: Z_{1}: 1\right]\right\} \cup\{[1: 0: 0],[0: 1: 0]\}$.

EXAMPLE 2.9. In the rank 2 case, $P \circ s$ is a well-defined map from $\mathbb{C} P^{1} \times \mathbb{C} P^{1}$ onto $\mathbb{C} P^{2}$, and $\mathbf{k}(\operatorname{ker}(\mathbf{P}))$ is equivalent to

$$
\left\{\lambda\left[\begin{array}{ll}
1 & 0 \\
0 & 1
\end{array}\right]: \lambda \in \mathbb{C}\right\}
$$

By Proposition 2.7, $\mathbf{P}$ is c-equivalent to

$$
\mathbf{Q}=\left[\begin{array}{cccc}
1 & 0 & 0 & -1 \\
0 & 1 & 0 & 0 \\
0 & 0 & 1 & 0
\end{array}\right]
$$

The singular locus of $Q \circ s$ is

$$
\Sigma_{2}=\left\{z_{0} w_{0}+z_{1} w_{1}=0\right\}
$$

and outside $\Sigma_{2}, Q \circ s$ is two-to-one:

$$
\begin{aligned}
(Q \circ s)\left(\left(\left[z_{0}: z_{1}\right],\left[w_{0}: w_{1}\right]\right)\right) & =\left[z_{0} w_{0}-z_{1} w_{1}: z_{0} w_{1}: z_{1} w_{0}\right] \\
& =(Q \circ s)\left(\left(\left[-w_{1}: w_{0}\right],\left[-z_{1}: z_{0}\right]\right)\right) .
\end{aligned}
$$

Let $\sigma: \mathbb{C} P^{1} \times \mathbb{C} P^{1} \rightarrow \mathbb{C} P^{1} \times \mathbb{C} P^{1}:\left(\left[z_{0}: z_{1}\right],\left[w_{0}: w_{1}\right]\right) \mapsto\left(\left[-w_{1}: w_{0}\right],\left[-z_{1}: z_{0}\right]\right)$, so $\sigma$ is a holomorphic involution such that $Q \circ s=Q \circ s \circ \sigma$, and its fixed point set is exactly $\Sigma_{2}$.

2.3. Real projective geometry. The maps to be introduced in this subsection are the inclusion

$$
\boldsymbol{\delta}_{m}: \mathbb{R}^{m+1} \rightarrow \mathbb{C}^{m+1}:\left(u_{0}, \ldots, u_{m}\right)^{T} \mapsto\left(u_{0}+0 i, \ldots, u_{m}+0 i\right)^{T}
$$


and the real linear involution of $\mathbb{C}^{m+1}$ defined by entrywise complex conjugation:

$$
\mathbf{C}_{m}: \mathbb{C}^{m+1} \rightarrow \mathbb{C}^{m+1}:\left(z_{0}, \ldots, z_{m}\right)^{T} \mapsto\left(\bar{z}_{0}, \ldots, \bar{z}_{m}\right)^{T}
$$

The image of $\boldsymbol{\delta}_{m}$ is exactly the fixed point set of $\mathbf{C}_{m}$. Both maps induce well-defined maps of projective spaces:

$$
\delta_{m}: \mathbb{R} P^{m} \rightarrow \mathbb{C} P^{m}, C_{m}: \mathbb{C} P^{m} \rightarrow \mathbb{C} P^{m} .
$$

As shown in $[6, \S 2.3]$, the image $\delta_{m}\left(\mathbb{R} P^{m}\right)$ is a smoothly embedded submanifold of $\mathbb{C} P^{m}$, and it is the fixed point set of the involution $C_{m}$.

Using the product map

$$
\delta_{\ell} \times \delta_{m}: \mathbb{R} P^{\ell} \times \mathbb{R} P^{m} \rightarrow \mathbb{C} P^{\ell} \times \mathbb{C} P^{m},
$$

the composition $s_{\mathbb{C}}^{\ell m} \circ\left(\delta_{\ell} \times \delta_{m}\right): \mathbb{R} P^{\ell} \times \mathbb{R} P^{m} \rightarrow \mathbb{C} P^{\ell m+\ell+m}$ is also a smooth embedding. It has the following form, for $u=\left[u_{0}: \cdots: u_{\ell}\right] \in \mathbb{R} P^{\ell}$ and $v=\left[v_{0}: \cdots: v_{m}\right] \in$ $\mathbb{R} P^{m}$ :

$$
(u, v) \mapsto\left[u_{0} v_{0}: u_{0} v_{1}: \cdots: u_{0} v_{m}: \cdots: u_{\ell} v_{0}: u_{\ell} v_{1}: \cdots: u_{\ell} v_{m}\right] .
$$

The image of $s_{\mathbb{C}}^{\ell m} \circ\left(\delta_{\ell} \times \delta_{m}\right)$ is the real Segre variety named in the title, and by the obvious equality $s_{\mathbb{C}}^{\ell m} \circ\left(\delta_{\ell} \times \delta_{m}\right)=\delta_{\ell m+\ell+m} \circ s_{\mathbb{R}}^{\ell m}$, the image is contained in a real projective space $\delta_{\ell m+\ell+m}\left(\mathbb{R} P^{\ell m+\ell+m}\right) \subseteq \mathbb{C} P^{\ell m+\ell+m}$. It will be convenient to abbreviate this map as $s_{\mathbb{C}}^{\ell m} \circ\left(\delta_{\ell} \times \delta_{m}\right)=s \circ(\delta \times \delta)$ when the dimensions are clear from context. Given a coefficient matrix $\mathbf{P}$, the composition $P \circ s \circ(\delta \times \delta): \mathbb{R} P^{\ell} \times \mathbb{R} P^{m} \rightarrow$ $\mathbb{C} P^{n}$ is smooth at points where it is well-defined, but it is not necessarily one-to-one or nonsingular. It is possible that $P \circ s \circ(\delta \times \delta)$ is well-defined, or an embedding, even if $P \circ s$ is neither. We will also use the conventions mentioned after Definition 2.1 about the domain and image of maps $P \circ s \circ(\delta \times \delta)$ when there are base points.

As mentioned in the introduction, the composition $P \circ s \circ(\delta \times \delta)$ is of the form

$$
(u, v) \mapsto\left[P_{0}: P_{1}: \cdots: P_{n}\right],
$$

with complex coefficients $p_{k}^{i, j}$ on quadratic terms in real variables:

$$
P_{k}=\sum_{j=0}^{m} \sum_{i=0}^{\ell} p_{k}^{i, j} u_{i} v_{j} .
$$

Maps of the form $P \circ s \circ(\delta \times \delta)$ will be the main objects of interest in subsequent sections. These real analytic parametrizations do not behave exactly like the complex analytic maps $P \circ s$. For instance, in contrast with Proposition 2.3, the matrices from Example 2.4 show that two maps $P \circ s \circ(\delta \times \delta), Q \circ s \circ(\delta \times \delta): \mathbb{R} P^{1} \times \mathbb{R} P^{1} \rightarrow \mathbb{C} P^{1}$ 
can be equal at every point in the domain, but $\mathbf{P}$ and $\mathbf{Q}$ are not related by scalar multiplication.

In the rest of this section, we will develop a notion of equivalence for coefficient matrices $\mathbf{P}$ which will be useful in studying the geometry of maps $P \circ s \circ(\delta \times \delta)$. The geometric idea is to consider real linear automorphisms of the domain $\mathbb{R} P^{\ell} \times \mathbb{R} P^{m}$, or equivalently, complex automorphisms of $\mathbb{C} P^{\ell} \times \mathbb{C} P^{m}$ that fix (as a set) the image of $\delta_{\ell} \times \delta_{m}$.

Definition 2.10. For fixed whole numbers $\ell, m, n$, two (complex) coefficient matrices $\mathbf{P}$ and $\mathbf{Q}$ are $r$-equivalent if there exist matrices $\mathbf{A}_{1} \in G L(\ell+1, \mathbb{C}), \mathbf{A}_{2} \in$ $G L(m+1, \mathbb{C}), \mathbf{B} \in G L(n+1, \mathbb{C})$ such that $\mathbf{A}_{1}=\mathbf{C}_{\ell} \circ \mathbf{A}_{1} \circ \mathbf{C}_{\ell}, \mathbf{A}_{2}=\mathbf{C}_{m} \circ \mathbf{A}_{2} \circ \mathbf{C}_{m}$, and for all $(\mathbf{z}, \mathbf{w}) \in\left(\mathbb{C}^{\ell+1} \backslash\{\mathbf{0}\}\right) \times\left(\mathbb{C}^{m+1} \backslash\{\mathbf{0}\}\right)$,

$$
\mathbf{Q}(\mathbf{s}((\mathbf{z}, \mathbf{w})))=\mathbf{B} \mathbf{P}\left(\mathbf{s}\left(\left(\mathbf{A}_{1} \mathbf{z}, \mathbf{A}_{\mathbf{2}} \mathbf{w}\right)\right)\right) \text {. }
$$

The following propositions are analogues of Theorem 2.15 and Corollary 2.16 in [6], and they have analogous proofs.

Proposition 2.11. Given coefficient matrices $\mathbf{P}$ and $\mathbf{Q}$, the following are equivalent:

1. $\mathbf{P}$ and $\mathbf{Q}$ are r-equivalent.

2. There exist $\mathbf{A}_{1} \in G L(\ell+1, \mathbb{C}), \mathbf{A}_{2} \in G L(m+1, \mathbb{C}), \mathbf{B} \in G L(n+1, \mathbb{C})$ such that $\mathbf{A}_{1}=\mathbf{C}_{\ell} \circ \mathbf{A}_{1} \circ \mathbf{C}_{\ell}, \mathbf{A}_{2}=\mathbf{C}_{m} \circ \mathbf{A}_{2} \circ \mathbf{C}_{m}$, and for all $(\mathbf{u}, \mathbf{v}) \in$ $\left(\mathbb{R}^{\ell+1} \backslash\{\mathbf{0}\}\right) \times\left(\mathbb{R}^{m+1} \backslash\{\mathbf{0}\}\right)$,

$$
\mathbf{Q}((\mathbf{s} \circ(\boldsymbol{\delta} \times \boldsymbol{\delta}))((\mathbf{u}, \mathbf{v})))=\mathbf{B} \mathbf{P}\left(\mathbf{s}\left(\left(\mathbf{A}_{1} \boldsymbol{\delta}(\mathbf{u}), \mathbf{A}_{2} \boldsymbol{\delta}(\mathbf{v})\right)\right)\right) .
$$

3. There exist $\underline{\mathbf{A}}_{1} \in G L(\ell+1, \mathbb{R}), \underline{\mathbf{A}}_{2} \in G L(m+1, \mathbb{R}), \mathbf{B} \in G L(n+1, \mathbb{C})$ such that for all $(\mathbf{u}, \mathbf{v}) \in\left(\mathbb{R}^{\ell+1} \backslash\{\mathbf{0}\}\right) \times\left(\mathbb{R}^{m+1} \backslash\{\mathbf{0}\}\right)$,

$$
\mathbf{Q}((\mathbf{s} \circ(\boldsymbol{\delta} \times \boldsymbol{\delta}))((\mathbf{u}, \mathbf{v})))=\mathbf{B} \mathbf{P}\left((\mathbf{s} \circ(\boldsymbol{\delta} \times \boldsymbol{\delta}))\left(\left(\underline{\mathbf{A}}_{1} \mathbf{u}, \underline{\mathbf{A}}_{2} \mathbf{v}\right)\right)\right) .
$$

4. There exist $\mathbf{A}_{1} \in G L(\ell+1, \mathbb{C})$ and $\mathbf{A}_{2} \in G L(m+1, \mathbb{C})$ such that $\mathbf{A}_{1}=$ $\mathbf{C}_{\ell} \circ \mathbf{A}_{1} \circ \mathbf{C}_{\ell}, \mathbf{A}_{2}=\mathbf{C}_{m} \circ \mathbf{A}_{2} \circ \mathbf{C}_{m}$ and

$$
\mathbf{k}(\operatorname{ker}(\mathbf{P}))=\mathbf{A}_{2}(\mathbf{k}(\operatorname{ker}(\mathbf{Q}))) \mathbf{A}_{1}^{T} .
$$

Proposition 2.12. Given coefficient matrices $\mathbf{P}$ and $\mathbf{Q}$, let $P$ and $Q$ be the induced projections. If $\mathbf{P}$ and $\mathbf{Q}$ are r-equivalent, then there exist automorphisms $A_{1} \in P G L(\ell+1, \mathbb{C}), A_{2} \in P G L(m+1, \mathbb{C}), B \in P G L(n+1, \mathbb{C})$ such that $A_{1}=$ $C_{\ell} \circ A_{1} \circ C_{\ell}, A_{2}=C_{m} \circ A_{2} \circ C_{m}$, and

$$
(Q \circ s)((z, w))=B\left((P \circ s)\left(\left(A_{1} z, A_{2} w\right)\right)\right)
$$


for all $(z, w) \in \mathbb{C} P^{\ell} \times \mathbb{C} P^{m}$ where both sides are defined. Conversely, if there exist $A_{1}$, $A_{2}$, and $B$ such that $A_{1}=C_{\ell} \circ A_{1} \circ C_{\ell}, A_{2}=C_{m} \circ A_{2} \circ C_{m}$, and $Q$ and $P$ satisfy the above equation at every point $(z, w) \in \mathbb{C} P^{\ell} \times \mathbb{C} P^{m}$, then $\mathbf{P}$ and $\mathbf{Q}$ are r-equivalent.

The relation $(1) \Longleftrightarrow$ (4) of Proposition 2.11 reduces the r-equivalence classification of coefficient matrices to the problem of finding the classes of complex subspaces of $M((m+1) \times(\ell+1), \mathbb{C})$, under left and right multiplication by invertible real matrices. This is the real equivalence of complex matrix pencils named in the title, and it will be the main tool for the classifications in Sections 4 and 5 .

In contrast with Proposition 2.6, the existence of $\underline{A}_{1}: \mathbb{R} P^{\ell} \rightarrow \mathbb{R} P^{\ell}, \underline{A}_{2}: \mathbb{R} P^{m} \rightarrow$ $\mathbb{R} P^{m}$ and $B: \mathbb{C} P^{n} \rightarrow \mathbb{C} P^{n}$ such that

$$
Q \circ s \circ(\delta \times \delta)=B \circ P \circ s \circ\left(\left(\delta \circ \underline{A}_{1}\right) \times\left(\delta \circ \underline{A}_{2}\right)\right): \mathbb{R} P^{\ell} \times \mathbb{R} P^{m} \rightarrow \mathbb{C} P^{n}
$$

at every point of $\mathbb{R} P^{\ell} \times \mathbb{R} P^{m}$ is, in general, not enough to establish the r-equivalence of $\mathbf{P}$ and $\mathbf{Q}$. The following example shows that such an equality of maps does not even imply c-equivalence of coefficient matrices.

EXAmple 2.13. Consider the case $\ell=m=1, n=0$, so a coefficient matrix $\mathbf{P}_{1 \times 4}=[1, i, i,-2]$ defines a parametric map

$$
P \circ s: \mathbb{C} P^{1} \times \mathbb{C} P^{1} \rightarrow \mathbb{C} P^{0}:\left(\left[z_{0}: z_{1}\right],\left[w_{0}: w_{1}\right]\right) \mapsto\left[z_{0} w_{0}-2 z_{1} w_{1}+i\left(z_{0} w_{1}+z_{1} w_{0}\right)\right] .
$$

Since the target space is just a point, the image of the map is not very interesting. However, the base point locus of $P \circ s$ is an interesting complex subvariety, corresponding to the intersection of the three-dimensional kernel of $\mathbf{P}$ and the image of the Segre map.

$$
\mathbf{k}(\operatorname{ker}(\mathbf{P}))=\left\{\lambda\left[\begin{array}{ll}
1 & 0 \\
i & 0
\end{array}\right]+\mu\left[\begin{array}{ll}
1 & i \\
0 & 0
\end{array}\right]+\nu\left[\begin{array}{ll}
2 & 0 \\
0 & 1
\end{array}\right]: \lambda, \mu, \nu \in \mathbb{C}\right\},
$$

and an element of this subspace of $M(2 \times 2, \mathbb{C})$ has rank $\leq 1$ if and only if $\lambda \mu+\lambda \nu+$ $\mu \nu+2 \nu^{2}=0$. The set of singular matrices is irreducible as an affine subvariety of $\mathbf{k}(\operatorname{ker}(\mathbf{P}))$. Another coefficient matrix of the same size is $\mathbf{Q}_{1 \times 4}=[1, i, i,-1]$, which defines a parametric map

$$
\begin{gathered}
Q \circ s:\left(\left[z_{0}: z_{1}\right],\left[w_{0}: w_{1}\right]\right) \mapsto\left[z_{0} w_{0}-z_{1} w_{1}+i\left(z_{0} w_{1}+z_{1} w_{0}\right)\right]=\left[\left(z_{0}+i z_{1}\right)\left(w_{0}+i w_{1}\right)\right] . \\
\mathbf{k}(\operatorname{ker}(\mathbf{Q}))=\left\{\lambda\left[\begin{array}{ll}
1 & 0 \\
i & 0
\end{array}\right]+\mu\left[\begin{array}{ll}
1 & i \\
0 & 0
\end{array}\right]+\nu\left[\begin{array}{ll}
1 & 0 \\
0 & 1
\end{array}\right]: \lambda, \mu, \nu \in \mathbb{C}\right\}
\end{gathered}
$$

and an element of this subspace of $M(2 \times 2, \mathbb{C})$ has rank $\leq 1$ if and only if $\lambda \mu+\lambda \nu+$ $\mu \nu+\nu^{2}=(\lambda+\nu)(\mu+\nu)=0$. This set of singular matrices is not irreducible. The 
equivalence automorphism of the space $M(2 \times 2, \mathbb{C}), \mathbf{S} \mapsto \mathbf{A}_{2} \mathbf{S} \mathbf{A}_{1}^{T}$ as in Proposition 2.7, is linear and rank-preserving. So, there can be no equivalence transformation taking the subspace $\mathbf{k}(\operatorname{ker}(\mathbf{Q}))$, which contains a pair of planes of singular matrices, to $\mathbf{k}(\operatorname{ker}(\mathbf{P}))$, which does not. By Proposition 2.7, $\mathbf{P}$ and $\mathbf{Q}$ are not c-equivalent matrices.

However, it is straightforward to see that both $P \circ s \circ(\delta \times \delta)$ and $Q \circ s \circ(\delta \times \delta)$ are well-defined at every point of $\mathbb{R} P^{1} \times \mathbb{R} P^{1}$, so they define the same (constant) map.

3. Equivalence of parametrizations. In the next two sections, we will consider maps of the form $P \circ s \circ(\delta \times \delta): \mathbb{R} P^{\ell} \times \mathbb{R} P^{m} \rightarrow \mathbb{C} P^{n}$, for specific choices of $\ell, m, n$. Recall that coefficient matrices have size $(n+1) \times(\ell+1)(m+1)$, and are full rank, with complex scalar multiples of a matrix $\mathbf{P}$ defining exactly the same projection $P$. So, the parameter space of projection matrices is a dense open subset of $\mathbb{C} P^{(\ell+1)(m+1)(n+1)-1}$, which has real dimension $2(\ell+1)(m+1)(n+1)-2$. The group acting on the matrix space, whose orbits are the r-equivalence classes, is $P G L(\ell+1, \mathbb{R}) \times P G L(m+1, \mathbb{R}) \times P G L(n+1, \mathbb{C})$, which has real dimension $\left((\ell+1)^{2}-1\right)+\left((m+1)^{2}-1\right)+2\left((n+1)^{2}-1\right)$. The difference between these two dimensions is the expected number of real moduli:

$$
\begin{aligned}
\mathcal{M}(\ell, m, n) & =2(\ell+1)(m+1)(n+1)-2-\left(\ell^{2}+2 \ell+m^{2}+2 m+2 n^{2}+4 n\right) \\
& =2 \ell m n+2 \ell m+2 \ell n+2 m n-\ell^{2}-m^{2}-2 n^{2}-2 n .
\end{aligned}
$$

However, the calculations of the next section will show that this dimension count is too naïve. For example, when $\ell=m=1$ and $n=2, P G L(2, \mathbb{R}) \times P G L(2, \mathbb{R}) \times P G L(3, \mathbb{C})$, a 22-dimensional group, acts on the 22-dimensional space of coefficient matrices, so $\mathcal{M}(1,1,2)=0$, but there will still be a one-parameter family of inequivalent matrices. Instead of trying to solve the r-equivalence classification problem for all $(\ell, m, n)$, the next two sections will cover only the simplest interesting cases: $(\ell, m, n)=(1,1,2)$, and $(2,1,4)$. We will find the real equivalence classes of complex matrix pencils, to get a representative of each of the r-equivalence classes of coefficient matrices, and to see how the algebraic invariants of $\mathbf{P}$ correspond to geometric properties of the maps $P \circ s \circ(\delta \times \delta)$ and $P \circ s$.

It can happen, in some examples, that the image of $\delta \times \delta$ may contain base points of $P \circ s$, in which case $P \circ s \circ(\delta \times \delta)$ is not defined on all of $\mathbb{R} P^{\ell} \times \mathbb{R} P^{m}$. Other examples will show some of the distinguishing geometric features that the image $(P \circ s \circ(\delta \times \delta))\left(\mathbb{R} P^{\ell} \times \mathbb{R} P^{m}\right)$ may have: differential-topological singularities, or a locus of self-intersection. As remarked earlier, such points do not occur in the image of $s \circ(\delta \times \delta)$, but they could occur after the projection by $P$.

In addition to the differential topology of maps $P \circ s \circ(\delta \times \delta)$, it will be important to consider their interaction with the complex structure on the target space $\mathbb{C} P^{n}$. 
A real submanifold $M\left(\operatorname{dim}_{\mathbb{R}}=m\right)$ of a complex manifold $\left(\operatorname{dim}_{\mathbb{C}}=n\right.$ with complex structure operator $J$ on the tangent bundle) satisfies the following property at all points $x: \operatorname{dim}_{\mathbb{C}}\left(T_{x} M \cap J T_{x} M\right) \geq \max \{0, m-n\}$. If $M$ is in general position, then that dimension achieves its minimum $(0$ or $m-n)$ at most points $x$ (forming a dense open subset of $M$ ). The exceptional points $x \in M$, where the tangent space contains a complex ( $J$-invariant) subspace of greater dimension than this minimum are called $C R$ singular points.

The image of $s \circ(\delta \times \delta): \mathbb{R} P^{\ell} \times \mathbb{R} P^{m} \rightarrow \mathbb{C} P^{\ell m+\ell+m}$ is a real submanifold, and at each point, the tangent space contains no complex lines, so it is called totally real. There could be CR singular points after the projection by $P$, and the locus of such points will be another interesting feature to see when classifying maps $P \circ s \circ(\delta \times \delta)$. If $(u, v)$ is an element of $\mathbb{R} P^{\ell} \times \mathbb{R} P^{m}$, and $P \circ s$ is nonsingular at $(\delta \times \delta)((u, v)) \in$ $\mathbb{C} P^{\ell} \times \mathbb{C} P^{m}$, then $P \circ s$ will be a complex analytic diffeomorphism of a neighborhood of $(\delta \times \delta)((u, v))$ onto a neighborhood in $(P \circ s)\left(\mathbb{C} P^{\ell} \times \mathbb{C} P^{m}\right)$, and since $(\delta \times \delta)\left(\mathbb{R} P^{\ell} \times\right.$ $\left.\mathbb{R} P^{m}\right)$ is totally real near $(\delta \times \delta)((u, v))$, the image $(P \circ s \circ(\delta \times \delta))\left(\mathbb{R} P^{\ell} \times \mathbb{R} P^{m}\right)$ will also be totally real near $(P \circ s \circ(\delta \times \delta))((u, v))$. So, the only candidates for CR singularities in the image of $P \circ s \circ(\delta \times \delta)$ will be images of singular points of $P \circ s$, and this phenomenon will be observed in the next sections.

We will, further, be considering the projective-geometric properties of the map $P \circ s \circ(\delta \times \delta)$, in particular, how the image of the map intersects complex lines in $\mathbb{C} P^{n}$. The CR singular points, where the complex lines are tangent to the image, are an example of this, but the geometric invariants of these intersections under the action of $P G L(n+1, \mathbb{C})$ will also be useful in distinguishing different equivalence classes of totally real embeddings.

4. Ruled surfaces in the complex projective plane. In the case $\ell=m=1$, $n=2$, a $3 \times 4$ matrix $\mathbf{P}$ with rank 3 determines a map $P \circ s \circ(\delta \times \delta): \mathbb{R} P^{1} \times \mathbb{R} P^{1} \rightarrow \mathbb{C} P^{2}$, of the form

$$
\left(\left[u_{0}: u_{1}\right],\left[v_{0}: v_{1}\right]\right) \mapsto\left[P_{0}: P_{1}: P_{2}\right],
$$

where each $P_{k}$ has complex coefficients:

$$
P_{k}=p_{k}^{0,0} u_{0} v_{0}+p_{k}^{0,1} u_{0} v_{1}+p_{k}^{1,0} u_{1} v_{0}+p_{k}^{1,1} u_{1} v_{1} .
$$

The images of such bihomogeneous quadratic parametrizations will generally be surfaces doubly ruled by real lines, although this must be taken in the sense of circles or lines as in the inversive geometry of a complex projective line. It will turn out (Corollary 4.10) that there is always some affine neighborhood in which the double ruling is actually by straight lines.

The solution of the c-equivalence problem for $3 \times 4$ coefficient matrices was recalled in Section 2.2. Under the smaller group, where only real changes of variables in the 
domain are allowed, there will be more equivalence classes. The rank 1 case, where $P \circ s$ is undefined at one point, will split into four cases, depending on whether this point is in the image of $\delta \times \delta$ (Example 4.2), $I d_{\mathbb{C} P^{1}} \times \delta$ (Example 4.3), $\delta \times I d_{\mathbb{C} P^{1}}$ (Example 4.4), or none of these (Example 4.8). The rank 2 case will split into some one-parameter families.

The following theorem gives the real equivalence classes of one-dimensional matrix pencils. Its list of normal forms resembles, but is not exactly the same as the results from [16], which considered the real congruence classes of complex symmetric matrix subspaces, and [5], which considered the Hermitian congruence classes of subspaces of $M(2 \times 2, \mathbb{C})$. For example, the complex pencils spanned by $\left[\begin{array}{ll}1 & 0 \\ 0 & 1\end{array}\right]$ and $\left[\begin{array}{cc}1 & 0 \\ 0 & -1\end{array}\right]$ are in the same real equivalence class, but not the same real congruence class. One step of the proof will use the classification of [16].

THEOREM 4.1. If $\mathbf{K}$ is a non-zero matrix in $M(2 \times 2, \mathbb{C})$, then there is exactly one matrix in the list below equal to $\lambda \mathbf{A}_{2} \mathbf{K} \mathbf{A}_{1}^{T}$ for some nonsingular real matrices $\mathbf{A}_{1}, \mathbf{A}_{2}$, and non-zero complex scalar $\lambda$.

$$
\begin{aligned}
& \text { 1. }\left[\begin{array}{ll}
1 & 0 \\
0 & 0
\end{array}\right] ; \\
& \text { 2. }\left[\begin{array}{ll}
1 & i \\
0 & 0
\end{array}\right] ; \\
& \text { 3. }\left[\begin{array}{ll}
1 & 0 \\
i & 0
\end{array}\right] ; \\
& \text { 4. }\left[\begin{array}{cc}
0 & 1 / 2 \\
1 / 2 & i
\end{array}\right] ; \\
& \text { 5. }\left[\begin{array}{cc}
1 & 0 \\
0 & \alpha
\end{array}\right], \alpha=\cos (\theta)+i \sin (\theta), 0 \leq \theta \leq \frac{\pi}{2} ; \\
& \text { 6. }\left[\begin{array}{cc}
-i t^{2} & 1 \\
1 & i
\end{array}\right], 0<t \leq 1 .
\end{aligned}
$$

Proof. Let $\mathbf{K} \in M(2 \times 2, \mathbb{C})$, with real and imaginary parts:

$$
\mathbf{K}=\left[\begin{array}{cc}
a+i b & c+i d \\
e+i f & g+i h
\end{array}\right]=\operatorname{Re}(\mathbf{K})+i \operatorname{Im}(\mathbf{K})
$$

For the first of two main cases, suppose $\operatorname{det}(\operatorname{Re}(\lambda \mathbf{K}))=0$ for all $\lambda=x+i y \in \mathbb{C}$. This implies that the equation

$$
\operatorname{det}(\operatorname{Re}(\mathbf{K})) x^{2}+\operatorname{det}(\operatorname{Im}(\mathbf{K})) y^{2}+(-a h-b g+f c+e d) x y=0
$$

holds for all $x, y$, so its coefficients, $\operatorname{det}(\operatorname{Re}(\mathbf{K})), \operatorname{det}(\operatorname{Im}(\mathbf{K})),-a h-b g+f c+e d$, are all zero. If $\operatorname{Re}(\mathbf{K})$ is the zero matrix, then $\mathbf{K}$ is a complex scalar multiple of a real 
rank 1 matrix, which is in the same real equivalence class as case (1) of the theorem. If $\operatorname{Re}(\mathbf{K}) \neq 0$, then $\mathbf{K}$ is in the same real equivalence class as

$$
\left[\begin{array}{cc}
1+i b & i d \\
i f & i h
\end{array}\right]
$$

where the entries $b, d, f, h$ may have changed, but we follow a convention of using the same letters as place-holders. Equation (4.1) implies $h=0$ and $d f=0$. If $d=f=0$, $\mathbf{K}$ is in case (1) again. When $f=0$ and $d \neq 0$,

$$
\left[\begin{array}{cc}
1+i b & i d \\
0 & 0
\end{array}\right]\left[\begin{array}{cc}
1 & 0 \\
-\frac{b}{d} & \frac{1}{d}
\end{array}\right]=\left[\begin{array}{ll}
1 & i \\
0 & 0
\end{array}\right]
$$

and when $d=0$ and $f \neq 0$,

$$
\left[\begin{array}{cc}
1 & -\frac{b}{f} \\
0 & \frac{1}{f}
\end{array}\right]\left[\begin{array}{cc}
1+i b & 0 \\
i f & 0
\end{array}\right]=\left[\begin{array}{cc}
1 & 0 \\
i & 0
\end{array}\right],
$$

yielding cases (2) and (3) of the theorem. It is a straightforward calculation to check that cases (1), (2), (3) are in three different real equivalence classes.

The second main case is when some complex scalar multiple of $\mathbf{K}$ has real part with rank 2. By multiplication on one side by the inverse of that real part, the complex line spanned by $\mathbf{K}$ is in the same real equivalence class as the span of

$$
\left[\begin{array}{ll}
1 & 0 \\
0 & 1
\end{array}\right]+i\left[\begin{array}{ll}
b & d \\
f & h
\end{array}\right]
$$

and a real similarity transformation fixes the real part and puts the imaginary part into its real Jordan canonical form [13], exactly one of the following cases:

$$
\begin{aligned}
& {\left[\begin{array}{ll}
1 & 0 \\
0 & 1
\end{array}\right]+i\left[\begin{array}{ll}
b & 0 \\
0 & h
\end{array}\right], b \geq h} \\
& {\left[\begin{array}{ll}
1 & 0 \\
0 & 1
\end{array}\right]+i\left[\begin{array}{ll}
b & 1 \\
0 & b
\end{array}\right],} \\
& {\left[\begin{array}{ll}
1 & 0 \\
0 & 1
\end{array}\right]+i\left[\begin{array}{cc}
b & d \\
-d & b
\end{array}\right], d>0}
\end{aligned}
$$

In the case of the diagonal canonical form, a complex re-scaling by $(1+i b)^{-1}$ puts $\mathbf{K}$ in the form $\left[\begin{array}{ll}1 & 0 \\ 0 & \alpha\end{array}\right]$, where $\alpha$ can be equal to 1 or any complex number with a nonzero imaginary part. Left multiplication by $\left[\begin{array}{cc}1 & 0 \\ 0 & |\alpha|^{-1}\end{array}\right]$ puts entry $\alpha$ on the unit 
circle. Left and right multiplication by $\left[\begin{array}{ll}0 & 1 \\ 1 & 0\end{array}\right]$, and another complex re-scaling, switches $\alpha$ to $\frac{1}{\alpha}$, and a left multiplication can also switch $\alpha$ to $-\alpha$; using these as necessary gives case (5) of the theorem. It is easy to check that for each $\alpha$, this rank 2 pencil is not in the same real equivalence class as any of the other representatives in case (5), nor in any of the rank 1 classes.

In the repeated eigenvalue case, the calculation

$$
\left[\begin{array}{cc}
0 & \frac{1}{2 \sqrt{1+b^{2}}} \\
\sqrt{1+b^{2}} & \frac{-b}{\sqrt{1+b^{2}}}
\end{array}\right]\left[\begin{array}{cc}
1 & \frac{i}{1+i b} \\
0 & 1
\end{array}\right]\left[\begin{array}{cc}
\frac{1}{2 \sqrt{1+b^{2}}} & 0 \\
0 & \sqrt{1+b^{2}}
\end{array}\right]=\left[\begin{array}{cc}
0 & \frac{1}{2} \\
\frac{1}{2} & i
\end{array}\right]
$$

shows that any such pencil is in the same real equivalence class as case (4) of the theorem. It is easy to check that the matrix from case (4) is not in the same real equivalence class as any diagonal matrix, so the representatives in cases (4) and (5) are in distinct equivalence classes.

In the last case, where the imaginary part has non-real eigenvalues, consider the following calculation:

$$
\begin{aligned}
& \mathbf{A}_{2} \mathbf{K} \mathbf{A}_{1}^{T}=\left[\begin{array}{ll}
p & q \\
r & s
\end{array}\right]\left[\begin{array}{cc}
1+i b & i d \\
-i d & 1+i b
\end{array}\right]\left[\begin{array}{ll}
w & x \\
y & z
\end{array}\right] \\
& =\left[\begin{array}{ll}
w p+y q+i(w p b-w q d+y p d+y q b) & x p+z q+i(x p b-x q d+z p d+z q b) \\
w r+y s+i(w r b-w s d+y r d+y s b) & x r+z a+i(x r b-x s d+z r d+z s b)
\end{array}\right] .
\end{aligned}
$$

If the upper left entry of the product were zero, then $w p+y q=0$, and also $-w q+y p=$ 0 , since $d>0$. This is impossible, since the only real solutions of this system of equations are $\{w=0, y=0\}$ or $\{p=0, q=0\}$, both of which contradict the requirement that $\mathbf{A}_{2}$ and $\mathbf{A}_{1}$ are invertible. Analogous calculations show that the matrix $\mathbf{K}$ is not in the same real equivalence class as any matrix with any zero entries, and so $\mathbf{K}$ is not in cases (1) through (5) of the theorem.

However, $\mathbf{K}$ is in the same real equivalence class as a symmetric matrix, $\mathbf{K}^{\prime}$; for example,

$$
\begin{aligned}
\mathbf{K}^{\prime} & =\left[\begin{array}{cc}
d-b & d+b \\
1 & -1
\end{array}\right]\left[\begin{array}{cc}
1+i b & i d \\
-i d & 1+i b
\end{array}\right]\left[\begin{array}{cc}
d-b & 1 \\
-b-d & 1
\end{array}\right] \\
& =\left[\begin{array}{cc}
-4 b d-2 i d b^{2}-2 i d^{3} & 2 d \\
2 d & 2 i d
\end{array}\right] .
\end{aligned}
$$

According to the classification result of [16], there exist a real matrix $\mathbf{A}$, a complex number $\lambda$ and a real number $t \in(0,1]$, such that

$$
\lambda \mathbf{A} \mathbf{K}^{\prime} \mathbf{A}^{T}=\left[\begin{array}{cc}
-i t^{2} & 1 \\
1 & i
\end{array}\right]
$$


a matrix we will denote by $\mathbf{K}(t)$. The only such matrix with rank 1 is $\mathbf{K}(1)$. To prove the theorem, it remains only to show that for $s, t \in(0,1)$, if the pencils spanned by $\mathbf{K}(s)$ and $\mathbf{K}(t)$ are in the same real equivalence class, then $s=t$.

So, suppose there exist $\mathbf{L}, \mathbf{R} \in G L(2, \mathbb{R})$ and a non-zero complex number $x+i y$ so that $\mathbf{L} \mathbf{K}(t) \mathbf{R}=(x+i y) \mathbf{K}(s)$. Taking the real and imaginary parts of both sides implies

$$
\mathbf{L} \operatorname{Re}(\mathbf{K}(t)) \mathbf{R}=\operatorname{Re}((x+i y) \mathbf{K}(s)),
$$

or

$$
\mathbf{L}\left[\begin{array}{ll}
0 & 1 \\
1 & 0
\end{array}\right] \mathbf{R}=\operatorname{Re}((x+i y) \mathbf{K}(s))
$$

or

$$
\mathbf{L}=\operatorname{Re}((x+i y) \mathbf{K}(s)) \mathbf{R}^{-1}\left[\begin{array}{ll}
0 & 1 \\
1 & 0
\end{array}\right]
$$

and

$$
\mathbf{L} \operatorname{Im}(\mathbf{K}(t)) \mathbf{R}=\operatorname{Im}((x+i y) \mathbf{K}(s)),
$$

or

$$
\operatorname{Re}((x+i y) \mathbf{K}(s)) \mathbf{R}^{-1}\left[\begin{array}{ll}
0 & 1 \\
1 & 0
\end{array}\right] \operatorname{Im}(\mathbf{K}(t)) \mathbf{R}=\operatorname{Im}((x+i y) \mathbf{K}(s)),
$$

or

$$
\mathbf{R}^{-1}\left[\begin{array}{ll}
0 & 1 \\
1 & 0
\end{array}\right] \operatorname{Im}(\mathbf{K}(t)) \mathbf{R}=(\operatorname{Re}((x+i y) \mathbf{K}(s)))^{-1} \operatorname{Im}((x+i y) \mathbf{K}(s))
$$

or

$$
\mathbf{R}^{-1}\left[\begin{array}{cc}
0 & 1 \\
-t^{2} & 0
\end{array}\right] \mathbf{R}=\left[\begin{array}{cc}
y s^{2} & x \\
x & -y
\end{array}\right]^{-1}\left[\begin{array}{cc}
-x s^{2} & y \\
y & x
\end{array}\right]
$$

Since $\left[\begin{array}{cc}0 & 1 \\ -t^{2} & 0\end{array}\right]$ and the product on the RHS are similar, they have the same eigenvalues:

$$
\{ \pm i t\}=\left\{\frac{\left(1-s^{2}\right) x y \pm i s\left(x^{2}+y^{2}\right)}{x^{2}+y^{2} s^{2}}\right\}
$$

Because we are assuming $0<s<1$, the only way the real part of each of the eigenvalues can be zero is when $x=0$ or $y=0$. Then, comparing the imaginary 
parts, if $x=0$, then $t= \pm \frac{1}{s}$, and if $y=0$, then $t= \pm s$, so the only solution in $(0,1)$ is $t=s$.

In the following list of examples, we will consider the various geometric properties of maps of the form $Q \circ s \circ(\delta \times \delta)$, for each of the $r$-equivalence classes of matrices $\mathbf{Q}$, corresponding to the normal forms of the previous theorem.

The two cases where the kernel is spanned by a real matrix ((1), and (5) with $\theta=0$ ) represent projections where the center of projection is in $\delta_{3}\left(\mathbb{R} P^{3}\right) \subseteq \mathbb{C} P^{3}$, and so the image of $Q \circ s \circ(\delta \times \delta)$ is contained in a real projective plane, $\left(Q \circ \delta_{3}\right)\left(\mathbb{R} P^{3}\right)=$ $\delta_{2}\left(\mathbb{R} P^{2}\right) \subseteq \mathbb{C} P^{2}$ (see Figure 4$)$.

In the remaining cases, the center of projection is outside $\delta_{3}\left(\mathbb{R} P^{3}\right)$, and the image of $Q \circ s \circ(\delta \times \delta)$ is contained in $\left(Q \circ \delta_{3}\right)\left(\mathbb{R} P^{3}\right) \subseteq \mathbb{C} P^{2}$. In each of the examples, it will be easy to pick at least one complex affine neighborhood $U \subseteq \mathbb{C} P^{2}$ such that $U \cap\left(\left(Q \circ \delta_{3}\right)\left(\mathbb{R} P^{3}\right)\right)$ is a real affine 3 -space in $U$, allowing us to visualize $U \cap((Q \circ$ $\left.s \circ(\delta \times \delta))\left(\mathbb{R} P^{1} \times \mathbb{R} P^{1}\right)\right)$. While $Q \circ \delta_{3}: \mathbb{R} P^{3} \rightarrow \mathbb{C} P^{2}$ is one-to-one on some affine neighborhoods, it is not an immersion of $\mathbb{R} P^{3}$, since in each case there is a real line which is collapsed to a point.

It will also be useful to observe how complex projective lines in $\mathbb{C} P^{2}$ meet the surface, and the set $\left(Q \circ \delta_{3}\right)\left(\mathbb{R} P^{3}\right)$ that contains the surface. In a complex affine neighborhood $U$ whose intersection with $\left(Q \circ \delta_{3}\right)\left(\mathbb{R} P^{3}\right)$ is a real affine 3 -space, that 3 -space will contain a parallel family of complex affine lines, and every other complex line in $U$ will either be disjoint from the 3 -space, or will meet it transversely in a real affine line. In some other complex affine neighborhood $V$, those complex lines still look like complex lines, but $V \cap\left(\left(Q \circ \delta_{3}\right)\left(\mathbb{R} P^{3}\right)\right)$ may not be a real affine space. It contains a family of complex lines, but the previously mentioned transverse intersections with complex lines may transform from lines to circles or lines, since the action of $P G L(3, \mathbb{C})$ on $\mathbb{C} P^{2}$ restricts to a $P G L(2, \mathbb{C})$ action on each complex line.

Let $\mathbb{C} P^{2}$ have homogeneous coordinates $\left[Z_{0}: Z_{1}: Z_{2}\right]$, with $Z_{j}=X_{j}+i Y_{j}$.

EXAMPLE 4.2. The rank 1 matrix in case (1) of Theorem 4.1 has already appeared in Example 2.8; using the matrix $\mathbf{Q}$ from that example, the map

$$
Q \circ s \circ(\delta \times \delta): \mathbb{R} P^{1} \times \mathbb{R} P^{1} \rightarrow \mathbb{C} P^{2}:\left(\left[u_{0}: u_{1}\right],\left[v_{0}: v_{1}\right]\right) \mapsto\left[u_{0} v_{1}: u_{1} v_{0}: u_{1} v_{1}\right]
$$

has geometric properties analogous to those of $Q \circ s$. It is undefined at one point of the domain: the base point of $Q \circ s$ is an element of the image of $\delta \times \delta$. The image of $Q \circ s \circ(\delta \times \delta)$ is contained in $\delta_{2}\left(\mathbb{R} P^{2}\right) \subseteq \mathbb{C} P^{2}$, so $Q \circ s \circ(\delta \times \delta)$ is a smooth but singular map from a punctured torus to, but not onto, a real projective plane.

EXAMPLE 4.3. Another rank 1 matrix in Theorem 4.1 is case (2), where a repre- 
sentative coefficient matrix, i.e., a matrix with a kernel spanned by vec $\left(\left[\begin{array}{ll}1 & i \\ 0 & 0\end{array}\right]\right)$, is

$$
\mathbf{Q}=\left[\begin{array}{llll}
1 & 0 & i & 0 \\
0 & 1 & 0 & 0 \\
0 & 0 & 0 & 1
\end{array}\right]
$$

The induced map is

$$
Q \circ s \circ(\delta \times \delta):\left(\left[u_{0}: u_{1}\right],\left[v_{0}: v_{1}\right]\right) \mapsto\left[u_{0} v_{0}+i u_{1} v_{0}: u_{0} v_{1}: u_{1} v_{1}\right],
$$

which is defined on all of $\mathbb{R} P^{1} \times \mathbb{R} P^{1}$, but $Q \circ s$ has a base point at $([1: i],[1: 0]) \in$ $\mathbb{C} P^{1} \times \mathbb{C} P^{1}$. The singular locus of $Q \circ s$ is, as in Example 2.8,

$$
\Sigma_{1}=\left\{w_{1}\left(z_{0}+i z_{1}\right)=0\right\} \backslash\{([1: i],[1: 0])\} .
$$

The line $\left\{\left(\left[u_{0}: u_{1}\right],[1: 0]\right)\right\}$ is the intersection of the image of $\delta \times \delta$ with $\Sigma_{1}$, and it is mapped to a point: $Q \circ s \circ(\delta \times \delta):\left\{\left(\left[u_{0}: u_{1}\right],[1: 0]\right)\right\} \mapsto[1: 0: 0]$. Away from this line, $Q \circ s \circ(\delta \times \delta)$ is a totally real immersion.

$Q \circ s \circ(\delta \times \delta)$ restricts to a parametric map $\mathbb{R}^{2} \rightarrow \mathbb{R}^{3}$, in the $\{([u: 1],[v: 1])\}$, $\left\{\left[X_{0}+i Y_{0}: X_{1}: 1\right]\right\}$ neighborhoods: $(u, v) \mapsto\left(X_{0}, Y_{0}, X_{1}\right)=(u v, v, u)$. The image is a hyperbolic paraboloid in the $X_{0}, Y_{0}, X_{1} 3$-space (Figure 4.1). All of the complex lines contained in the $X_{0}, Y_{0}, X_{1} 3$-space are of the form $\left\{X_{1}=c\right\}=\left\{\left[Z_{0}: c Z_{2}: Z_{2}\right]\right\}$; each meets the surface in a straight line (from the real ruling). In $\mathbb{C} P^{2}$, all these complex lines meet at infinity, at the point $[1: 0: 0]$, which is the differential-topological singularity of the surface.

To get an idea of the shape of the singularity, consider a restriction to a different affine neighborhood: $Q \circ s \circ(\delta \times \delta):([1: u],[1: v]) \mapsto[1+i u: v: u v]=$ $\left[1: \frac{v}{1+i u}: \frac{u v}{1+i u}\right]$, or $(u, v) \mapsto\left(X_{1}, Y_{1}, X_{2}, Y_{2}\right)=\left(\frac{v}{1+u^{2}}, \frac{-u v}{1+u^{2}}, \frac{u v}{1+u^{2}}, \frac{-u^{2} v}{1+u^{2}}\right)$. The image in the $X_{1}, Y_{1}, X_{2}, Y_{2}$-space satisfies the implicit equations $X_{1} Y_{2}+X_{2}^{2}=Y_{1}+X_{2}=0$, so it is a cone contained in a 3 -space. The complex lines in the real 3-space $Y_{1}+X_{2}=0$ are of the form $Z_{1}+i Z_{2}=r$, for $r \in \mathbb{R}$, and they meet the cone in a set of circles, collapsing to a point at the vertex. These circles are one of the two real rulings of the surface; since they are contained in complex lines, they are projectively equivalent to some of the straight lines observed in the original affine neighborhood.

EXAMPLE 4.4. The rank 1 matrix in case (3) of Theorem 4.1 has a representative coefficient matrix

$$
\mathbf{P}=\left[\begin{array}{llll}
1 & i & 0 & 0 \\
0 & 0 & 1 & 0 \\
0 & 0 & 0 & 1
\end{array}\right]
$$




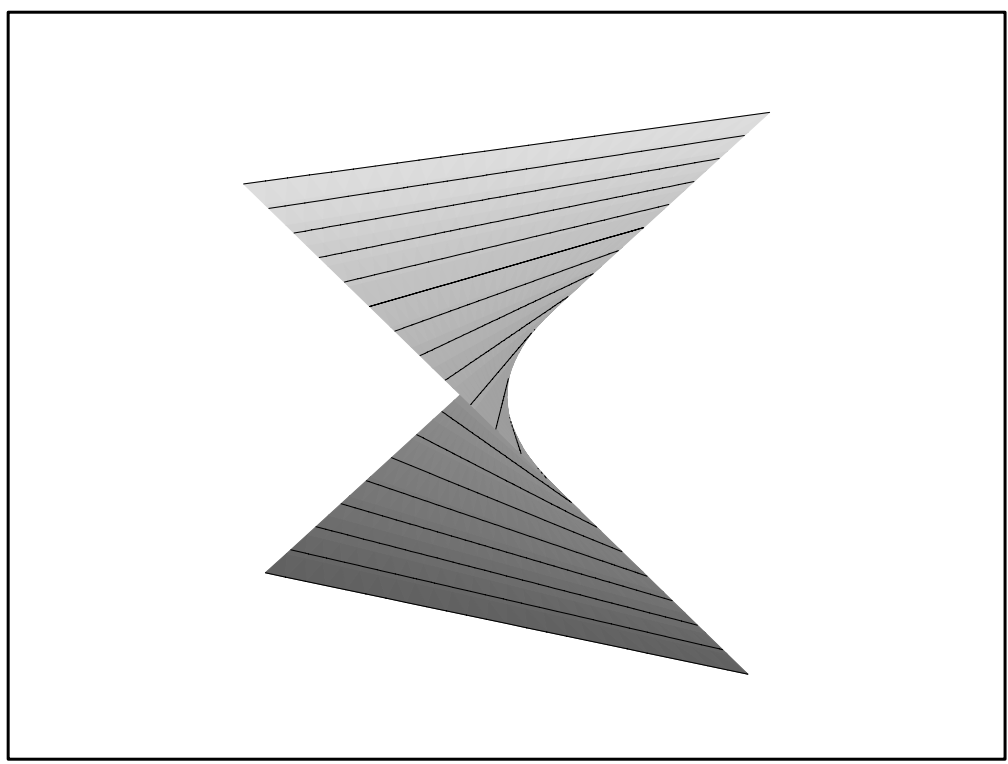

FIG. 4.1. A hyperbolic paraboloid, meeting complex lines in real lines, as in Examples 4.3 and 4.4 .

and the induced map is

$$
P \circ s \circ(\delta \times \delta):\left(\left[u_{0}: u_{1}\right],\left[v_{0}: v_{1}\right]\right) \mapsto\left[u_{0} v_{0}+i u_{0} v_{1}: u_{1} v_{0}: u_{1} v_{1}\right] .
$$

If $\varphi: \mathbb{C} P^{1} \times \mathbb{C} P^{1} \rightarrow \mathbb{C} P^{1} \times \mathbb{C} P^{1}$ is the holomorphic involution $\varphi:(z, w) \mapsto(w, z)$, then $\varphi$ fixes, as a set, the image of $\delta \times \delta$, and the matrix $\mathbf{P}$ is related to the matrix $\mathbf{Q}$ in Example 4.3 by the equation $P \circ s=Q \circ s \circ \varphi$. We can conclude that $P \circ s \circ(\delta \times \delta)$ and $Q \circ s \circ(\delta \times \delta)$ have exactly the same image, and the maps differ only in which line, from the first or second $\mathbb{R} P^{1}$ factor, is mapped to a point.

ExAmplE 4.5. The exceptional rank 2 matrix $\left[\begin{array}{cc}0 & 1 / 2 \\ 1 / 2 & i\end{array}\right]$ in case (4) of Theorem 4.1 has a representative coefficient matrix

$$
\mathbf{Q}=\left[\begin{array}{cccc}
1 & 0 & 0 & 0 \\
0 & 1 & -1 & 0 \\
0 & 0 & 2 & i
\end{array}\right]
$$

The induced map is

$$
Q \circ s \circ(\delta \times \delta):\left(\left[u_{0}: u_{1}\right],\left[v_{0}: v_{1}\right]\right) \mapsto\left[u_{0} v_{0}: u_{0} v_{1}-u_{1} v_{0}: 2 u_{1} v_{0}+i u_{1} v_{1}\right]
$$

The singular locus of $Q \circ s$ is

$$
\Sigma_{2}=\left\{2 z_{0} w_{0}+i\left(z_{0} w_{1}+z_{1} w_{0}\right)=0\right\}
$$


and the involution $\sigma$ is given by

$$
\sigma:\left(\left[z_{0}: z_{1}\right],\left[w_{0}: w_{1}\right]\right) \mapsto\left(\left[w_{0}: 2 i w_{0}-w_{1}\right],\left[z_{0}: 2 i z_{0}-z_{1}\right]\right) .
$$

One real line in $\delta_{3}\left(\mathbb{R} P^{3}\right)$ is projected by $Q$ to a point: $Q:\left\{\left[0: u_{1}: u_{1}: u_{3}\right]\right\} \mapsto[0:$ $0: 1]$, and this line meets the image of $s \circ(\delta \times \delta)$ exactly once, at $(s \circ(\delta \times \delta))(([0$ : $1],[0: 1]))=[0: 0: 0: 1]$.

The image of $\delta \times \delta$ in $\mathbb{C} P^{1} \times \mathbb{C} P^{1}$ intersects $\Sigma_{2}$ in exactly one point, ([0:1], [0:1]), so we can conclude that on the complement of that point, $Q \circ s \circ(\delta \times \delta)$ is a totally real immersion. The same point, $([0: 1],[0: 1])$, is also the only point of intersection of the image of $\delta \times \delta$ with the image of $\sigma \circ(\delta \times \delta)$, and it is fixed by $\sigma$, so we can further conclude that $Q \circ s \circ(\delta \times \delta)$ is a one-to-one map. It remains only to check the behavior of $Q \circ s \circ(\delta \times \delta)$ at the point ([0:1], [0:1]), and in fact it has a singularity there as a smooth map, where the rank of the (real) Jacobian drops to 1 at that point.

The map $Q \circ s \circ(\delta \times \delta)$ restricts to a parametric map $\mathbb{R}^{2} \rightarrow \mathbb{R}^{3}$, in the $\{([1: u],[1:$ $v])\},\left\{\left[1: X_{1}: X_{2}+i Y_{2}\right]\right\}$ neighborhoods: $(u, v) \mapsto\left(X_{1}, X_{2}, Y_{2}\right)=(v-u, 2 u, u v)$. The image of $Q \circ s \circ(\delta \times \delta)$ is the hyperbolic paraboloid $4 Y_{2}=X_{2}\left(2 X_{1}+X_{2}\right)$ in this neighborhood (Figure 4.2). All of the complex lines contained in the $X_{1}, X_{2}, Y_{2}$ 3 -space are of the form $\left\{X_{1}=c\right\}=\left\{\left[Z_{0}: c Z_{0}: Z_{2}\right]\right\}$; each meets the surface in a parabola, and none is a tangent plane, so the surface is totally real in this neighborhood. In $\mathbb{C} P^{2}$, all these complex lines meet at infinity, at the point $[0: 0: 1]$, which is the differential-topological singularity of the surface. The point $[0: 0: 1]$ is also the cusp singularity of each of the parabola-shaped intersections, which have a cardioid shape in a complex projective line $[6,8]$.

The two lines outside the $\{([1: u],[1: v])\}$ neighborhood in the domain are mapped to straight lines, $\left(\left[u_{0}: u_{1}\right],[0: 1]\right) \mapsto\left[0: u_{0}: i u_{1}\right]$ and $\left([0: 1],\left[v_{0}: v_{1}\right]\right) \mapsto[0:$ $\left.-v_{0}: 2 v_{0}+i v_{1}\right]$, which meet at $[0: 0: 1]$. There is another affine neighborhood where the surface looks like a hyperboloid of one sheet in $\mathbb{R}^{3}$ (Figure 4.3). The complex lines contained in that $\mathbb{R}^{3}$ meet the surface in parabolas, except for one complex line which meets it in a pair of parallel lines.

A restriction to a different affine neighborhood will show the singularity: $Q \circ s \circ$ $(\delta \times \delta):([u: 1],[v: 1]) \mapsto[u v: u-v: 2 v+i]=\left[\frac{u v}{2 v+i}: \frac{u-v}{2 v+i}: 1\right]$, or $(u, v) \mapsto$ $\left(X_{0}, Y_{0}, X_{1}, Y_{1}\right)=\left(\frac{2 u v^{2}}{4 v^{2}+1}, \frac{-u v}{4 v^{2}+1}, \frac{2 v(u-v)}{4 v^{2}+1}, \frac{v-u}{4 v^{2}+1}\right)$. The image in the $X_{0}, Y_{0}, X_{1}, Y_{1}$ 4 -space satisfies the implicit equations

$$
\begin{aligned}
& 0=X_{1}^{2}+2 X_{1}^{3}+4 X_{0} X_{1} Y_{1}+2 X_{1} Y_{1}^{2}+4 Y_{0} Y_{1}^{2}, \\
& 0=X_{0} X_{1}+2 X_{0} X_{1}^{2}+4 X_{0}^{2} Y_{1}+4 Y_{0}^{2} Y_{1}+2 X_{0} Y_{1}^{2}, \\
& 0=X_{0}^{2}+2 X_{0}^{2} X_{1}+4 X_{0}^{2} Y_{0}+4 Y_{0}^{3}+2 X_{0} Y_{0} Y_{1}, \\
& 0=X_{1} Y_{0}-X_{0} Y_{1} .
\end{aligned}
$$




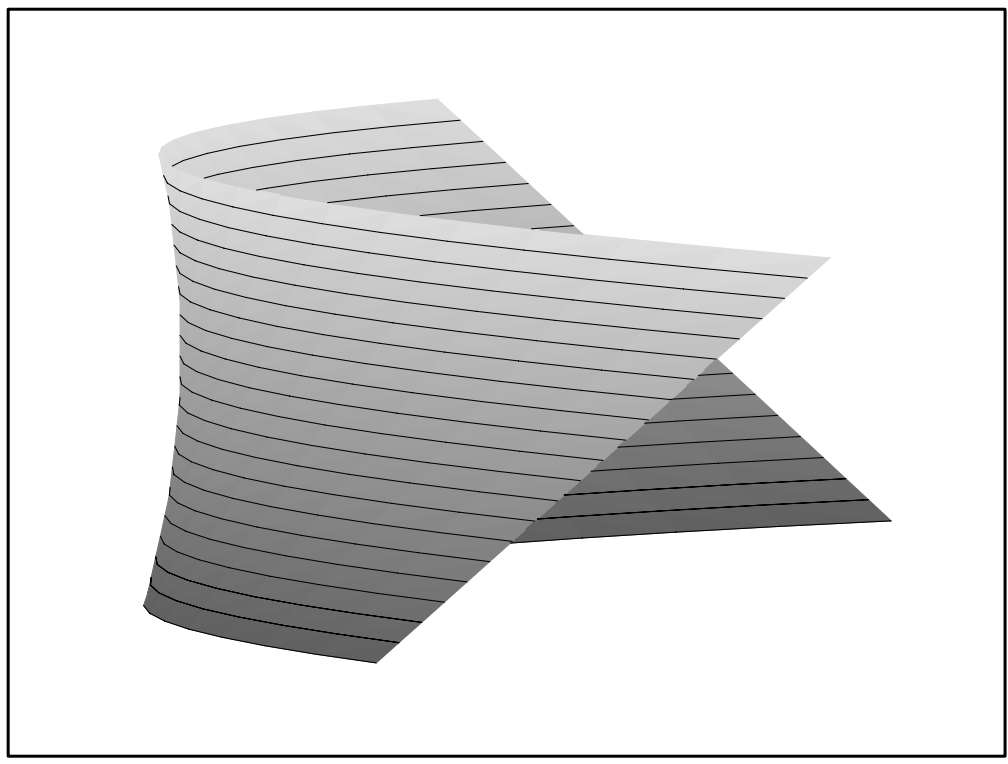

FIG. 4.2. A hyperbolic paraboloid, meeting complex lines in parabolas, as in Example 4.5.

A computation checks that these equations define a two-dimensional real variety with a singularity only at the origin.

EXAMPLE 4.6. The matrix in case (5), with $\alpha=1$, has a real representative coefficient matrix, which has already appeared in Example 2.9. The map $Q \circ s \circ(\delta \times \delta)$ is defined on all of $\mathbb{R} P^{1} \times \mathbb{R} P^{1}$. Its singular locus is a real curve in the domain, and it is two-to-one outside this locus. Its image is contained in, but not equal to, $\delta_{2}\left(\mathbb{R} P^{2}\right) \subseteq \mathbb{C} P^{2}$, so it can be considered as a real projection of the real Segre variety to a real projective plane (Figure 4.4), and as the $\theta \rightarrow 0^{+}$limiting case of the complex projections in the next example.

EXAMPLE 4.7. The remaining matrices in case (5), with $\alpha=e^{i \theta}, 0<\theta \leq \frac{\pi}{2}$, correspond to representative coefficient matrices of the form:

$$
\mathbf{Q}_{\alpha}=\left[\begin{array}{cccc}
\alpha & 0 & 0 & -1 \\
0 & 1 & 0 & 0 \\
0 & 0 & 1 & 0
\end{array}\right] .
$$

For each $\alpha$, the induced map is

$$
Q_{\alpha} \circ s \circ(\delta \times \delta):\left(\left[u_{0}: u_{1}\right],\left[v_{0}: v_{1}\right]\right) \mapsto\left[\alpha u_{0} v_{0}-u_{1} v_{1}: u_{0} v_{1}: u_{1} v_{0}\right] .
$$

The singular locus of $Q_{\alpha} \circ s$ is

$$
\Sigma_{2}=\left\{\alpha z_{0} w_{0}+z_{1} w_{1}=0\right\},
$$


Electronic Journal of Linear Algebra ISSN 1081-3810

A publication of the International Linear Algebra Society

ELA

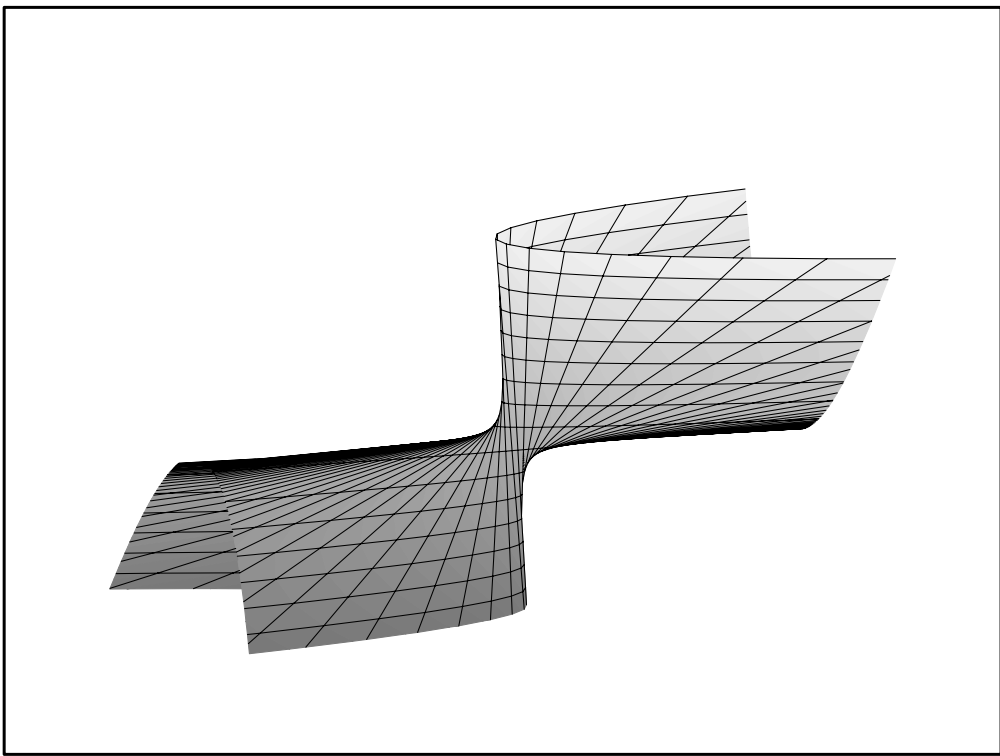

FIG. 4.3. A hyperboloid, meeting complex lines in parabolas and a pair of lines, as in Example 4.5 .

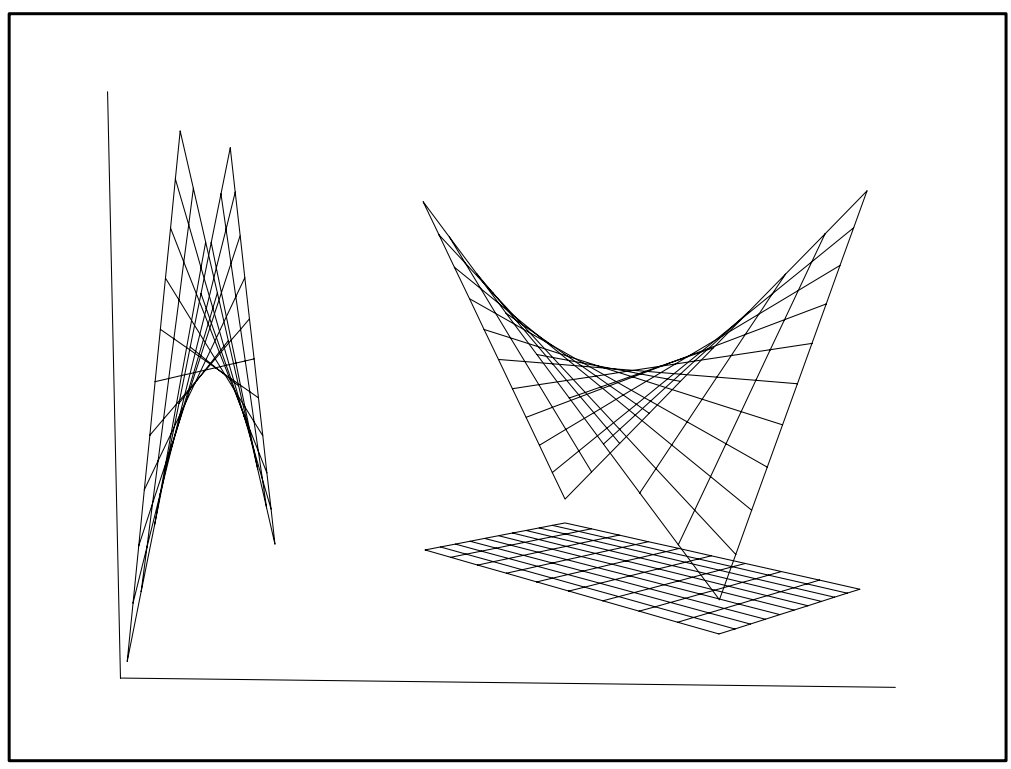

FIG. 4.4. The real Segre surface in $\mathbb{R}^{3}$ (center), with its two types of real projections, as in Examples 4.2 (below) and 4.6 (left). 
and the involution $\sigma$ is given by

$$
\sigma:\left(\left[z_{0}: z_{1}\right],\left[w_{0}: w_{1}\right]\right) \mapsto\left(\left[-w_{1}: \alpha w_{0}\right],\left[-z_{1}: \alpha z_{0}\right]\right) .
$$

One real line in $\delta_{3}\left(\mathbb{R} P^{3}\right)$ is projected by $Q_{\alpha}$ to a point: $Q_{\alpha}:\left\{\left[u_{0}: 0: 0: u_{3}\right]\right\} \mapsto[1:$ $0: 0]$, and this line meets the image of $s \circ(\delta \times \delta)$ twice, at $(s \circ(\delta \times \delta))(([0: 1],[0:$ $1]))=[0: 0: 0: 1]$, and $(s \circ(\delta \times \delta))(([1: 0],[1: 0]))=[1: 0: 0: 0]$.

The image of $\delta \times \delta$ in $\mathbb{C} P^{1} \times \mathbb{C} P^{1}$ intersects $\Sigma_{2}$ in exactly two points, ([1:0], [0:1]) and $([0: 1],[1: 0])$, so we can conclude that on the complement of these two points, $Q_{\alpha} \circ s \circ(\delta \times \delta)$ is a totally real immersion. The image of $\delta \times \delta$ meets the image of $\sigma \circ(\delta \times \delta)$ in exactly four points: the two points $([1: 0],[0: 1])$ and $([0: 1],[1: 0])$ are fixed by $\sigma$, and the two points $([0: 1],[0: 1])$ and $([1: 0],[1: 0])$ are interchanged by $\sigma$, so we can further conclude that $Q_{\alpha} \circ s \circ(\delta \times \delta)$ is one-to-one except for exactly one double point, where

$$
\left(Q_{\alpha} \circ s \circ(\delta \times \delta)\right)(([0: 1],[0: 1]))=\left(Q_{\alpha} \circ s \circ(\delta \times \delta)\right)(([1: 0],[1: 0]))=[1: 0: 0] .
$$

Restricting $Q_{\alpha} \circ s \circ(\delta \times \delta)$ to some affine neighborhoods shows what's going on near the singular points of $Q \circ s$. Considering

$$
Q_{\alpha} \circ s \circ(\delta \times \delta):([1: u],[v: 1]) \mapsto[\alpha v-u: 1: u v]
$$

as a map from $\mathbb{R}^{2}$ to the $X_{0}, Y_{0}, X_{2} 3$-space, this restriction is a smooth embedding, and its tangent plane at $\left(X_{0}, Y_{0}, X_{2}\right)=(0,0,0)$ is the complex line $X_{2}=0$. This is the only complex tangent in this affine neighborhood, the surface being totally real at the other points. For $\alpha=a+i b$ with $b>0$, the surface is a hyperbolic paraboloid (Figure 4.5), given by the equation

$$
X_{2}=\frac{1}{b^{2}}\left(a Y_{0}^{2}-b X_{0} Y_{0}\right)=\frac{1}{4 b^{2}}\left(2 a Z_{0} \bar{Z}_{0}-\bar{\alpha} Z_{0}^{2}-\alpha \bar{Z}_{0}^{2}\right) .
$$

The ratio of coefficients $\beta=\left|\frac{-\alpha}{2 a}\right|$ is the well-known Bishop invariant, and it is a local biholomorphic invariant of the surface near the CR singularity [4]. So, there is a one-to-one correspondence between $\alpha=e^{i \theta}, 0<\theta \leq \pi / 2$, and all the values of the Bishop invariant in the set $\left(\frac{1}{2}, \infty\right]$.

All of the complex lines contained in this 3-space are of the form $\left\{X_{2}=c\right\}=$ $\left\{\left[Z_{0}: Z_{1}: c Z_{1}\right]\right\}$; one is the complex tangent plane, but the rest meet the surface in hyperbolas, with a constant eccentricity. In $\mathbb{C} P^{2}$, all these complex lines meet at infinity, at the point $[1: 0: 0]$, which is the point of self-intersection of the surface. The point $[1: 0: 0]$ is also the self-intersection point of each of the hyperbola-shaped intersections, which have a lemniscate shape in a complex projective line $[6,8]$.

In another affine neighborhood, the parametric map restricts to

$$
Q_{\alpha} \circ s \circ(\delta \times \delta):([u: 1],[1: v]) \mapsto[\alpha u-v: u v: 1] .
$$




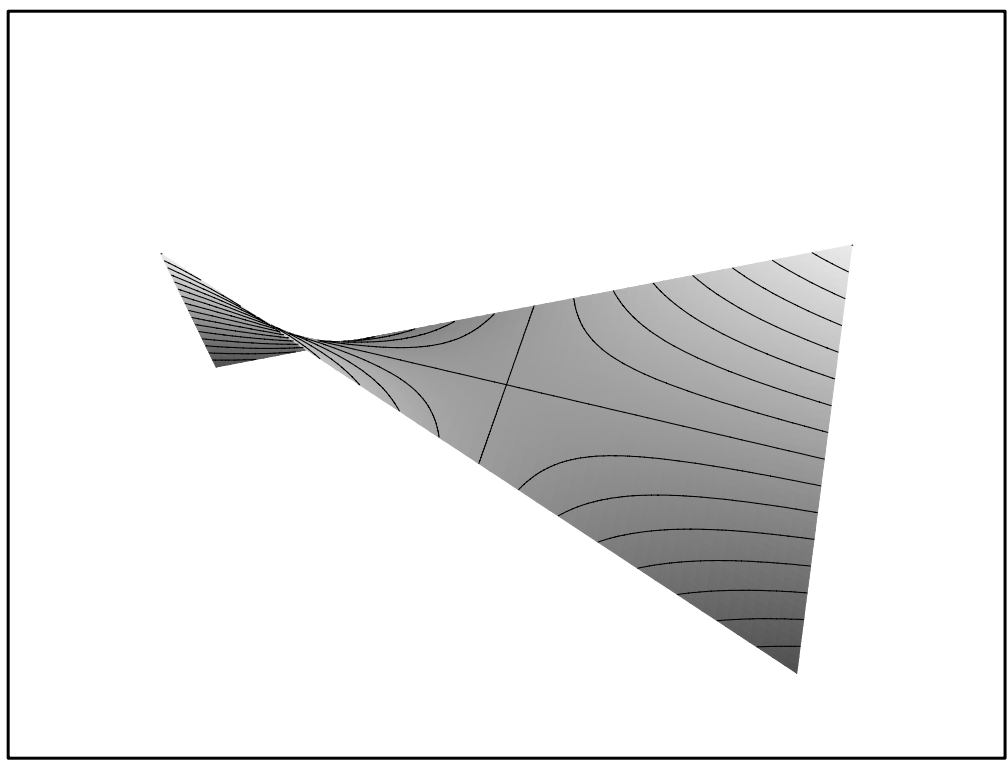

FIG. 4.5. A hyperbolic paraboloid, meeting complex lines in hyperbolas, and tangent to one complex line, as in Example 4.7.

The image is another hyperbolic paraboloid in the $X_{0}, Y_{0}, X_{1}$ space, with a CR singularity at the origin, a point that was not an element of the previous affine neighborhood. The two singularities have the same Bishop invariant.

There are other complex affine neighborhoods whose intersection with the surface looks like a one-sheeted hyperboloid, showing both CR singular points (Figure 4.6).

It is interesting that the r-equivalence class of $\mathbf{Q}_{\alpha}$ can be detected by a local biholomorphic invariant at one of the distinguished points in the image of $Q_{\alpha} \circ s \circ$ $(\delta \times \delta)$.

EXAMPLE 4.8. The remaining rank one equivalence class in Theorem 4.1 is case (6), with $t=1$. A representative coefficient matrix is

$$
\mathbf{Q}_{1}=\left[\begin{array}{cccc}
1 & 0 & 0 & 1 \\
0 & 1 & -1 & 0 \\
0 & 0 & 1 & i
\end{array}\right]
$$

The induced map is

$$
Q_{1} \circ s \circ(\delta \times \delta):\left(\left[u_{0}: u_{1}\right],\left[v_{0}: v_{1}\right]\right) \mapsto\left[u_{0} v_{0}+u_{1} v_{1}: u_{0} v_{1}-u_{1} v_{0}: u_{1} v_{0}+i u_{1} v_{1}\right] .
$$

The composite $Q_{1} \circ s$ has a base point at $([1: i],[1: i])$, and its singular locus is

$$
\Sigma_{1}=\left\{\left(z_{0}+i z_{1}\right)\left(w_{0}+i w_{1}\right)=0\right\} \backslash\{([1: i],[1: i])\} .
$$




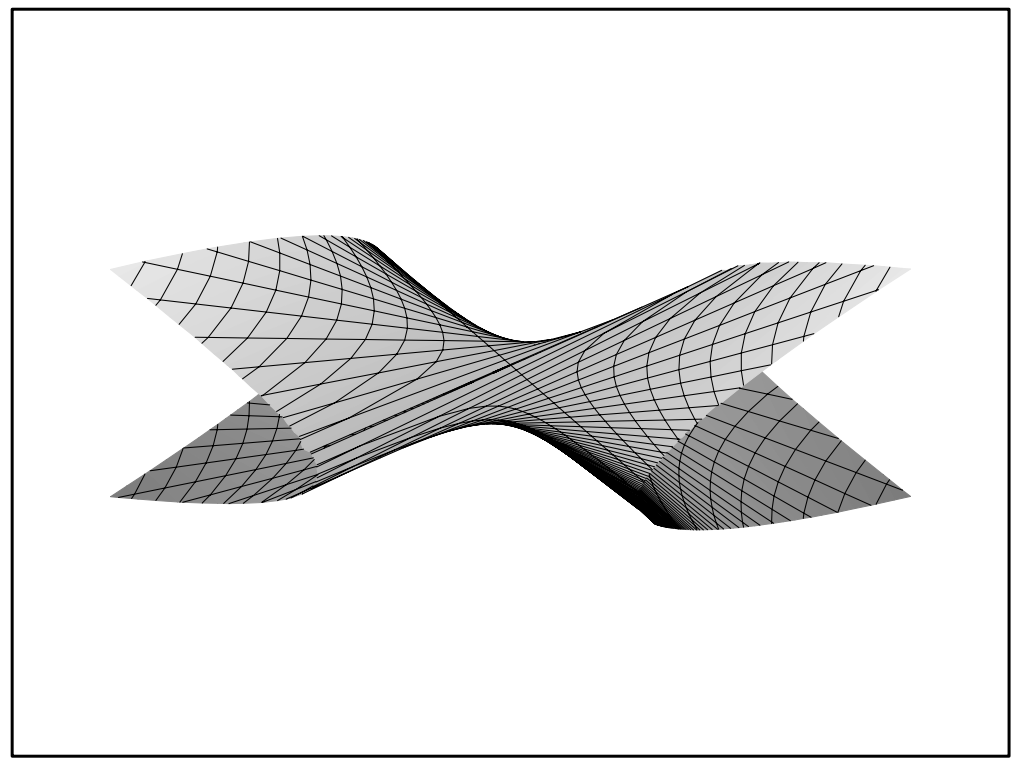

FIG. 4.6. A hyperboloid, meeting complex lines in hyperbolas, and tangent to two complex lines, as in Example 4.7.

One real line in $\delta_{3}\left(\mathbb{R} P^{3}\right)$ is projected by $Q_{1}$ to a point: $Q_{1}:\left\{\left[u_{0}: u_{1}: u_{1}:-u_{0}\right]\right\} \mapsto$ $[0: 0: 1]$, but this line does not meet the image of $s \circ(\delta \times \delta)$.

The image of $\delta \times \delta$ in $\mathbb{C} P^{1} \times \mathbb{C} P^{1}$ is disjoint from $\Sigma_{1}$, and $Q_{1} \circ s \circ(\delta \times \delta)$ is defined at every point of $\mathbb{R} P^{1} \times \mathbb{R} P^{1}$, so we can conclude that $Q_{1} \circ s \circ(\delta \times \delta)$ is a totally real embedding.

The intersection of the image of $Q_{1} \circ s \circ(\delta \times \delta)$ with the $Z_{0}=1$ neighborhood is contained in the real 3-space with coordinates $X_{1}, X_{2}, Y_{2}$, and is given by the equation $X_{2}^{2}+X_{1} X_{2}+Y_{2}^{2}-Y_{2}=0$. This is a hyperboloid of one sheet, with no tangent planes parallel to the complex line $X_{1}=0$. In fact, the only complex lines in that 3 -space are of the form $X_{1}=c$, and the intersection of the plane $X_{1}=c$ with the surface is a circle. Similarly, in the $Z_{1}=1$ neighborhood, the image is the hyperboloid $X_{2}^{2}+X_{2}+Y_{2}^{2}-X_{0} Y_{2}=0$ in the $X_{0}, X_{2}, Y_{2}$-space, whose intersections with the complex lines $X_{0}=c$ are circles. The real lines which rule the surface are also equal to the intersection of the surface with complex lines. These two complex affine neighborhoods cover the image of $Q_{1} \circ s \circ(\delta \times \delta)$, which does not contain the point $[0: 0: 1] \in \mathbb{C} P^{2}$.

This surface has the property that if it meets a complex line in a curve, then that curve is a circle or line, and this property is invariant under the action of $P G L(3, \mathbb{C})$. 
EXAMPLE 4.9. The last family of equivalence classes appearing in Theorem 4.1 is in case (6), with $0<t<1$. Representative coefficient matrices are of the form:

$$
\mathbf{Q}_{t}=\left[\begin{array}{cccc}
1 & 0 & 0 & t^{2} \\
0 & 1 & -1 & 0 \\
0 & 0 & 1 & i
\end{array}\right]
$$

For each $t$, the induced map is

$$
Q_{t} \circ s \circ(\delta \times \delta):\left(\left[u_{0}: u_{1}\right],\left[v_{0}: v_{1}\right]\right) \mapsto\left[u_{0} v_{0}+t^{2} u_{1} v_{1}: u_{0} v_{1}-u_{1} v_{0}: u_{1} v_{0}+i u_{1} v_{1}\right] .
$$

The singular locus of $Q_{t} \circ s$ is

$$
\Sigma_{2}=\left\{z_{0} w_{0}+i z_{0} w_{1}+i z_{1} w_{0}-t^{2} z_{1} w_{1}=0\right\},
$$

and the involution $\sigma$ is given by

$$
\sigma:\left(\left[z_{0}: z_{1}\right],\left[w_{0}: w_{1}\right]\right) \mapsto\left(\left[-i w_{0}+t^{2} w_{1}: w_{0}+i w_{1}\right],\left[-i z_{0}+t^{2} z_{1}: z_{0}+i z_{1}\right]\right) .
$$

One real line in $\delta_{3}\left(\mathbb{R} P^{3}\right)$ is projected by $Q_{t}$ to a point: $Q_{t}:\left\{\left[u_{0}: u_{1}: u_{1}:-u_{0} / t^{2}\right]\right\} \mapsto$ $[0: 0: 1]$, but this line does not meet the image of $s \circ(\delta \times \delta)$.

The image of $\delta \times \delta$ in $\mathbb{C} P^{1} \times \mathbb{C} P^{1}$ is disjoint from $\Sigma_{2}$, so we can conclude that $Q_{t} \circ s \circ(\delta \times \delta)$ is a totally real immersion. The image of $\delta \times \delta$ is also disjoint from the image of $\sigma \circ(\delta \times \delta)$, so we can further conclude that $Q_{t} \circ s \circ(\delta \times \delta)$ is one-to-one, and a totally real embedding.

The intersection of the image of $Q_{t} \circ s \circ(\delta \times \delta)$ with the $Z_{0}=1$ neighborhood is contained in the real 3-space with coordinates $X_{1}, X_{2}, Y_{2}$, and is given by the equation $X_{2}^{2}+X_{1} X_{2}+t^{2} Y_{2}^{2}-Y_{2}=0$ (Figure 4.7). This is a hyperboloid of one sheet, with no tangent planes parallel to the complex line $X_{1}=0$. All of the complex lines in that 3-space have the form $X_{1}=c$, and each intersects the surface in an ellipse with eccentricity $\sqrt{1-t^{2}}$, independent of $c$. Similarly, in the $Z_{1}=1$ neighborhood, the image is the hyperboloid $X_{2}^{2}+X_{2}+t^{2} Y_{2}^{2}-X_{0} Y_{2}=0$ in the $X_{0}, X_{2}, Y_{2}$-space, whose intersections with the complex lines $X_{0}=c$ are ellipses with eccentricity $\sqrt{1-t^{2}}$. These two complex affine neighborhoods cover the image of $Q_{t} \circ s \circ(\delta \times \delta)$, which does not contain the point $[0: 0: 1] \in \mathbb{C} P^{2}$.

It was remarked in [6] and proved in [8] that the eccentricity of an ellipse in a complex projective line is a complex projective $(P G L(2, \mathbb{C}))$ invariant, so that number is a $P G L(3, \mathbb{C})$ invariant of these surfaces, also. Any complex projective line meeting the surface in a curve will meet either in a circle or line (from the real ruling), or in a curve projectively equivalent to an ellipse, with a unique eccentricity.

Examples 4.8 and 4.9 show that for $0<t \leq 1$, the value of $t$, and therefore the r-equivalence class of the matrix $\mathbf{Q}_{t}$, can be detected by looking at the image of 


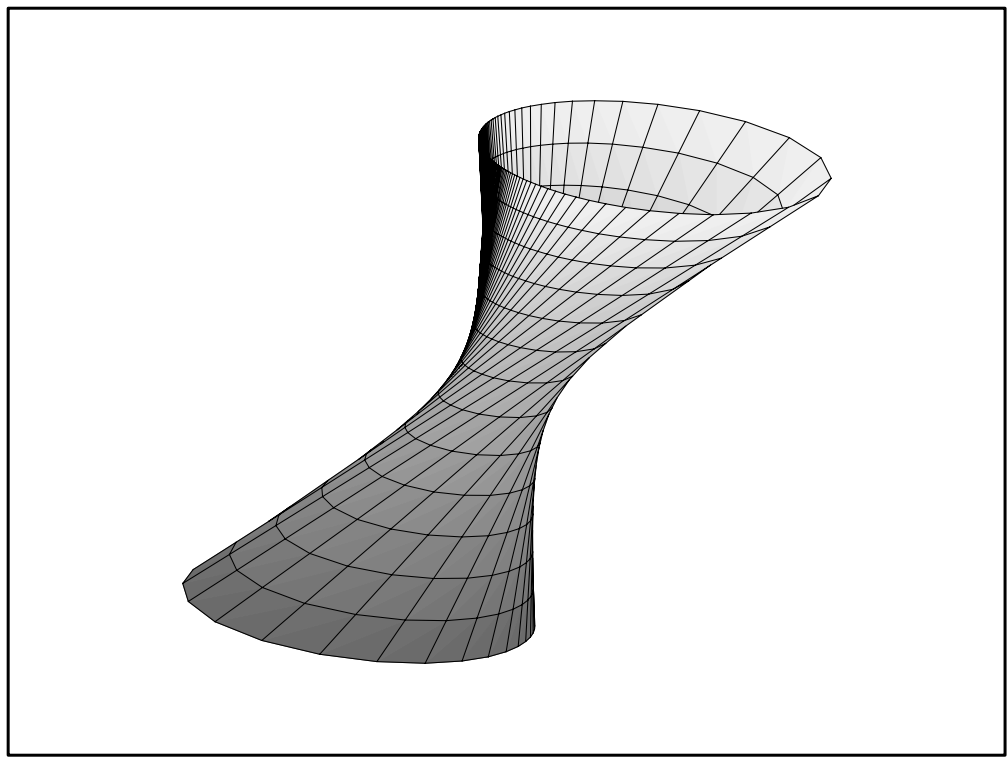

FIG. 4.7. A hyperboloid, meeting complex lines in ellipses, as in Example 4.9.

$Q_{t} \circ s \circ(\delta \times \delta)$. In fact, these surfaces can be distinguished in a local way by looking at a neighborhood of any point and how it intersects all the complex lines through that point. However, these surfaces cannot be distinguished by local biholomorphic invariants; since they are totally real and real analytic, they are locally biholomorphically equivalent.

The following corollaries of Theorem 4.1 summarize some of the observations from the previous examples.

Corollary 4.10. Let $V_{0}$ be the affine neighborhood $\left\{Z_{0}=1\right\}$. Given a coefficient matrix $\mathbf{P}_{3 \times 4}$, there exists $B \in P G L(3, \mathbb{C})$ and a real affine hyperplane $H \subseteq V_{0}$ such that the intersection of the image $(B \circ P \circ s \circ(\delta \times \delta))\left(\mathbb{R} P^{1} \times \mathbb{R} P^{1}\right)$ and $V_{0}$ is contained in a doubly ruled real affine quadric surface in $H$ (a plane, a hyperbolic paraboloid, or a hyperboloid of one sheet).

The next two corollaries strengthen the converse part of Proposition 2.12 in the $\ell=m=1, n=2$ case.

Corollary 4.11. Given coefficient matrices $\mathbf{P}_{3 \times 4}$ and $\mathbf{Q}_{3 \times 4}$, if there exist $A_{1}$, $A_{2} \in P G L(2, \mathbb{C})$, and $B \in P G L(3, \mathbb{C})$ such that $A_{1}=C_{1} \circ A_{1} \circ C_{1}, A_{2}=C_{1} \circ A_{2} \circ C_{1}$, and

$$
(Q \circ s)((\delta(u), \delta(v)))=B\left((P \circ s)\left(\left(A_{1}(\delta(u)), A_{2}(\delta(v))\right)\right)\right)
$$


at every point $(u, v) \in \mathbb{R} P^{1} \times \mathbb{R} P^{1}$ where both sides are defined, then $\mathbf{P}$ and $\mathbf{Q}$ are r-equivalent.

Corollary 4.12. Given coefficient matrices $\mathbf{P}_{3 \times 4}$ and $\mathbf{Q}_{3 \times 4}$, if there exists $B \in P G L(3, \mathbb{C})$ such that the images of $Q \circ s \circ(\delta \times \delta)$ and $B \circ P \circ s \circ(\delta \times \delta)$ are the same, then either $\mathbf{P}$ and $\mathbf{Q}$ are r-equivalent, or one falls in case (2) of Theorem 4.1 while the other falls in case (3).

5. Complex projections of the real Segre threefold. The next simplest case is $\ell=2, m=1, n=4$, corresponding to the Segre 3-manifold embedded in $\mathbb{C} P^{5}$, and its projections to $\mathbb{C} P^{4}$. Let $\mathbb{C} P^{4}$ have homogeneous coordinates $\left[Z_{0}: Z_{1}: Z_{2}\right.$ : $\left.Z_{3}: Z_{4}\right]$, with $Z_{j}=X_{j}+i Y_{j}$.

As in the previous section, the rational maps $P \circ s: \mathbb{C} P^{2} \times \mathbb{C} P^{1} \rightarrow \mathbb{C} P^{4}$ are easy to describe and classify, and there are only two c-equivalence classes of coefficient matrices $\mathbf{P}$. By Proposition 2.7, the c-equivalence class of $\mathbf{P}_{5 \times 6}$ is determined by the equivalence class of the complex subspace $\mathbf{k}(\operatorname{ker}(\mathbf{P}))$ in $M(2 \times 3, \mathbb{C})$. Since the only invariant under equivalence of $2 \times 3$ matrices is the rank [13], there are only two equivalence classes of one-dimensional subspaces of $M(2 \times 3, \mathbb{C})$ : the class of pencils spanned by a rank 1 matrix, and the class of pencils spanned by a rank 2 matrix. Geometrically, the two c-equivalence classes correspond to the two ways to project the complex Segre threefold in $\mathbb{C} P^{5}$ to $\mathbb{C} P^{4}$ : the center of projection can be either on the variety or off the variety.

EXAMPLE 5.1. In the rank 1 case, the map $P \circ s: \mathbb{C} P^{2} \times \mathbb{C} P^{1} \rightarrow \mathbb{C} P^{4}$ has exactly one base point; since $\mathbf{k}(\operatorname{ker}(\mathbf{P}))$ is in the image of $\mathbf{s}$, it is equivalent to

$$
\left\{\lambda\left[\begin{array}{lll}
1 & 0 & 0 \\
0 & 0 & 0
\end{array}\right]: \lambda \in \mathbb{C}\right\}
$$

By Proposition 2.7, $\mathbf{P}$ is c-equivalent to

$$
\mathbf{Q}=\left[\begin{array}{llllll}
0 & 1 & 0 & 0 & 0 & 0 \\
0 & 0 & 1 & 0 & 0 & 0 \\
0 & 0 & 0 & 1 & 0 & 0 \\
0 & 0 & 0 & 0 & 1 & 0 \\
0 & 0 & 0 & 0 & 0 & 1
\end{array}\right]
$$

The composite map

$$
Q \circ s:\left(\left[z_{0}: z_{1}: z_{2}\right],\left[w_{0}: w_{1}\right]\right) \mapsto\left[z_{0} w_{1}: z_{1} w_{0}: z_{1} w_{1}: z_{2} w_{0}: z_{2} w_{1}\right]
$$

has its base point at $([1: 0: 0],[1: 0])$. Its restriction to an affine neighborhood is one-to-one and nonsingular: $\left(\left[z_{0}: z_{1}: 1\right],[w: 1]\right) \mapsto\left[z_{0}: z_{1} w: z_{1}: w: 1\right]$, but one of the lines outside this neighborhood is mapped to a point $\mathcal{X},([1: 0: 0],[w: 1]) \mapsto[1:$ 
$0: 0: 0: 0]$. There is also a (punctured projective) plane that is mapped to a line $\mathcal{T}$, $\left(\left[z_{0}: z_{1}: z_{2}\right],[1: 0]\right) \mapsto\left[0: z_{1}: 0: z_{2}: 0\right]$. The singular locus can be described with a bihomogeneous expression:

$$
\Sigma_{3}=\left\{z_{1} w_{1}=z_{2} w_{1}=0\right\} \backslash\{([1: 0: 0],[1: 0])\} .
$$

The image of $Q \circ s$ is contained in the hypersurface $\left\{Z_{1} Z_{4}-Z_{2} Z_{3}=0\right\}$, but is not equal to it; for example, the hypersurface contains the plane $\left\{Z_{2}=Z_{4}=0\right\}$, but the image intersects this plane only in the previously noted point $\mathcal{X}$ and line $\mathcal{T}$.

Example 5.2. In the rank 2 case, $P \circ s$ is a well-defined map from $\mathbb{C} P^{2} \times \mathbb{C} P^{1}$ to $\mathbb{C} P^{4}$, and $\mathbf{k}(\operatorname{ker}(\mathbf{P}))$ is equivalent to

$$
\left\{\lambda\left[\begin{array}{lll}
0 & 1 & 0 \\
0 & 0 & 1
\end{array}\right]: \lambda \in \mathbb{C}\right\}
$$

By Proposition 2.7, $\mathbf{P}$ is c-equivalent to

$$
\mathbf{Q}=\left[\begin{array}{cccccc}
0 & 0 & 1 & 0 & 0 & -1 \\
1 & 0 & 0 & 0 & 0 & 0 \\
0 & 1 & 0 & 0 & 0 & 0 \\
0 & 0 & 0 & 1 & 0 & 0 \\
0 & 0 & 0 & 0 & 1 & 0
\end{array}\right]
$$

The singular locus of

$$
Q \circ s:\left(\left[z_{0}: z_{1}: z_{2}\right],\left[w_{0}: w_{1}\right]\right) \mapsto\left[z_{1} w_{0}-z_{2} w_{1}: z_{0} w_{0}: z_{0} w_{1}: z_{1} w_{1}: z_{2} w_{0}\right]
$$

is the curve

$$
\Sigma_{4}=\left\{z_{0}=z_{1} w_{0}+z_{2} w_{1}=0\right\} .
$$

On the subset $\left\{z_{0}=1\right\} \cong \mathbb{C}^{2} \times \mathbb{C} P^{1}, Q \circ s$ is one-to-one and non-singular. The restriction to the subset $\Theta=\left\{z_{0}=0\right\} \cong \mathbb{C} P^{1} \times \mathbb{C} P^{1}$,

$$
Q \circ s:\left(\left[0: z_{1}: z_{2}\right],\left[w_{0}: w_{1}\right]\right) \mapsto\left[z_{1} w_{0}-z_{2} w_{1}: 0: 0: z_{1} w_{1}: z_{2} w_{0}\right],
$$

is exactly as in Example 2.9, two-to-one except along the set $\Sigma_{4}$, and the image of this restriction is disjoint from $(Q \circ s)\left(\left\{z_{0}=1\right\}\right)$. The image of $Q \circ s$ is the complex projective hypersurface

$$
\left\{Z_{0} Z_{1} Z_{2}+Z_{2}^{2} Z_{4}-Z_{1}^{2} Z_{3}=0\right\}
$$

whose singular locus is the complex projective plane $\left\{Z_{1}=Z_{2}=0\right\}=(Q \circ s)(\Theta)$. The cubic hypersurface (5.1) is exactly the same as the one considered by $[2, \S 3.4$, Eq. (3.47)]. 
The main problem now is to find the r-equivalence classes of coefficient matrices $\mathbf{P}_{5 \times 6}$. As in the previous section, the strategy is to classify one-dimensional complex matrix pencils up to real equivalence, and to apply Proposition 2.11. We can also say something about the geometry of the maps $P \circ s \circ(\delta \times \delta)$ before doing the algebra. We again expect the rank 1 case to break into at least four r-equivalence classes, depending on how the image of $\delta \times \delta$ meets the base point and the singular locus of $P \circ s$ (it will turn out to be exactly four classes again). In the rank 2 case, we expect the generic situation to be that the three-dimensional image of $\delta \times \delta$ misses the singular locus $\Sigma_{4}$, so that $P \circ s \circ(\delta \times \delta)$ will be a totally real embedding. In general, the $\mathrm{CR}$ singular locus of a $(n-1)$-dimensional real submanifold of a $n$-dimensional complex manifold is expected to have real codimension 4 [5], and this is consistent with our expectation that most of the images of $\mathbb{R} P^{2} \times \mathbb{R} P^{1}$ will be totally real in $\mathbb{C} P^{4}$, and that $\mathrm{CR}$ singularities will occur only in exceptional cases.

To start with the linear algebra, we first consider the problem of finding representatives for pairs of real matrices $(\mathbf{C}, \mathbf{D})$, under simultaneous equivalence by real transformations. This is called by [11] the strict equivalence of matrix pencils $\mathbf{C}+\lambda \mathbf{D}$, with representatives in the Kronecker canonical form. The following list of canonical forms for the $2 \times 3$ case is adapted from [10], and we use notation similar to theirs for the cases. The important part of the proposition is that only real matrices are used, and some of the cases $(5 a, 5 b, 5 c)$ require the real Jordan normal form, as in the proof of Theorem 4.1.

Proposition 5.3. The list below includes all matrices of the specified form, with $\gamma, \delta, \epsilon, \zeta \in \mathbb{R}$ such that $\gamma \neq 0, \delta \neq 0, \gamma \geq \delta$ and $\epsilon>0$. Given $(\mathbf{C}, \mathbf{D}) \in$ $M(2 \times 3, \mathbb{R}) \times M(2 \times 3, \mathbb{R})$, there exists exactly one pair of matrices $(\mathbf{E}, \mathbf{F})$ from the list which is equal to $\left(\mathbf{A}_{2} \mathbf{C} \mathbf{A}_{1}, \mathbf{A}_{2} \mathbf{D} \mathbf{A}_{1}\right)$ for some $\mathbf{A}_{1} \in G L(3, \mathbb{R})$ and $\mathbf{A}_{2} \in G L(2, \mathbb{R})$. 


\begin{tabular}{|c|c|c|c|c|c|c|c|c|c|c|c|c|c|}
\hline & & \multicolumn{2}{|c|}{$\mathbf{E}$} & \multicolumn{3}{|c|}{$\mathbf{F}$} & & \multicolumn{3}{|c|}{$\mathbf{E}$} & \multicolumn{3}{|c|}{$\mathbf{F}$} \\
\hline \multirow{2}{*}{1} & & 1 & 0 & & & & \multirow{2}{*}{9} & & 0 & & 0 & 0 & \\
\hline & 0 & 0 & 1 & 0 & 1 & 0 & & & 0 & 1 & 0 & 0 & $\gamma$ \\
\hline \multirow{2}{*}{2} & 0 & 0 & 0 & 1 & 0 & 0 & \multirow{2}{*}{10} & 0 & 1 & 0 & 0 & 0 & 0 \\
\hline & 0 & 0 & 1 & 0 & 1 & 0 & & 0 & 0 & 1 & 0 & 1 & 0 \\
\hline \multirow{2}{*}{3} & 0 & 0 & 0 & 0 & 1 & 0 & \multirow{2}{*}{11} & 0 & 1 & 0 & 0 & 0 & 0 \\
\hline & 0 & 0 & 0 & 0 & 0 & 1 & & 0 & 0 & 1 & 0 & 0 & 0 \\
\hline \multirow{2}{*}{4} & 0 & 0 & 0 & 0 & 0 & 1 & \multirow{2}{*}{12} & 0 & 0 & 0 & 0 & 0 & 0 \\
\hline & 0 & 0 & 1 & 0 & 1 & 0 & & 0 & 0 & 1 & 0 & 0 & 0 \\
\hline \multirow{2}{*}{$5 a$} & 0 & 1 & 0 & 0 & $\gamma$ & 0 & \multirow{2}{*}{13} & 0 & 0 & 0 & 0 & 0 & 0 \\
\hline & 0 & 0 & 1 & 0 & 0 & $\delta$ & & 0 & 0 & 0 & 0 & 0 & 0 \\
\hline \multirow{2}{*}{$5 b$} & 0 & 1 & 0 & 0 & $\gamma$ & 1 & \multirow{2}{*}{$1^{\prime}$} & 0 & 1 & 0 & 1 & 0 & 0 \\
\hline & 0 & 0 & 1 & 0 & 0 & $\gamma$ & & 0 & 0 & 1 & 0 & 0 & $\gamma$ \\
\hline \multirow{2}{*}{$5 c$} & 0 & 1 & 0 & 0 & & $\epsilon$ & \multirow{2}{*}{$10^{\prime}$} & 0 & 1 & 0 & 0 & 0 & 0 \\
\hline & 0 & 0 & 1 & 0 & $-\epsilon$ & $\zeta$ & & 0 & 0 & 1 & 0 & 0 & $\gamma$ \\
\hline \multirow{2}{*}{6} & 0 & 1 & 0 & 1 & 0 & 0 & \multirow{2}{*}{$4^{\prime}$} & 0 & 0 & 0 & 0 & 1 & 0 \\
\hline & 0 & 0 & 1 & 0 & 0 & 0 & & 0 & 0 & 1 & 0 & 0 & $\gamma$ \\
\hline \multirow{2}{*}{7} & 0 & 0 & 0 & 0 & 1 & 0 & \multirow{2}{*}{$7^{\prime}$} & 0 & 0 & 0 & 0 & 0 & 0 \\
\hline & 0 & 0 & 1 & 0 & 0 & 0 & & 0 & 0 & 1 & 0 & 1 & 0 \\
\hline \multirow{2}{*}{8} & 0 & 0 & 0 & 0 & 0 & 1 & \multirow{2}{*}{$9^{\prime}$} & 0 & 0 & 0 & 0 & 0 & 1 \\
\hline & 0 & 0 & 0 & 0 & 0 & 0 & & 0 & 0 & 1 & 0 & 0 & 0 \\
\hline
\end{tabular}

It is also remarked in [10], and easy to check, that case 1 of the above proposition is the generic case; the set of matrix pairs $(\mathbf{C}, \mathbf{D})$ which are equivalent to the pair in case 1 is a dense open subset of $M(2 \times 3, \mathbb{R}) \times M(2 \times 3, \mathbb{R})$.

The next step in the r-equivalence classification is to find the real equivalence classes of one-dimensional complex subspaces of $M(2 \times 3, \mathbb{C})$. Proposition 5.3 and Theorem 4.1 will be used to prove the following result.

THEOREM 5.4. If $\mathbf{K}$ is a non-zero matrix in $M(2 \times 3, \mathbb{C})$, then there is exactly one matrix in the list below equal to $\lambda \mathbf{A}_{2} \mathbf{K} \mathbf{A}_{1}^{T}$ for some nonsingular real matrices $\mathbf{A}_{1}, \mathbf{A}_{2}$, and non-zero complex scalar $\lambda$.
1. $\left[\begin{array}{lll}0 & 1 & 0 \\ 0 & 0 & 0\end{array}\right]$;
2. $\left[\begin{array}{lll}0 & 1 & i \\ 0 & 0 & 0\end{array}\right]$;
3. $\left[\begin{array}{lll}0 & 1 & 0 \\ 0 & i & 0\end{array}\right]$;
4. $\left[\begin{array}{ccc}0 & 0 & 1 / 2 \\ 0 & 1 / 2 & i\end{array}\right]$; 


$$
\begin{aligned}
& \text { 5. }\left[\begin{array}{lll}
0 & 1 & 0 \\
0 & 0 & \alpha
\end{array}\right], \alpha=\cos (\theta)+i \sin (\theta), 0 \leq \theta \leq \frac{\pi}{2} \\
& \text { 6. }\left[\begin{array}{ccc}
0 & -i t^{2} & 1 \\
0 & 1 & i
\end{array}\right], 0<t \leq 1 \\
& \text { 7. }\left[\begin{array}{lll}
i & 1 & 0 \\
0 & 0 & 1
\end{array}\right] \\
& \text { 8. }\left[\begin{array}{lll}
i & 1 & 0 \\
0 & i & 1
\end{array}\right] .
\end{aligned}
$$

Proof. Let $\mathbf{K} \in M(2 \times 3, \mathbb{C})$, with real and imaginary parts: $\mathbf{K}=\mathbf{C}+i \mathbf{D}$. By Proposition 5.3, there exists a real equivalence transformation taking $\mathbf{K}$ to $\mathbf{E}+i \mathbf{F}$, for some real pair $(\mathbf{E}, \mathbf{F})$ in the proposition's list of canonical forms. Since $\mathbf{K}$ is non-zero, we can ignore the proposition's case 13 .

For the first of two main parts of the proof, suppose $\mathbf{K}$ has rank 1. Since the rank is invariant under real equivalence, we only need to inspect the list of canonical forms to find pairs $(\mathbf{E}, \mathbf{F})$ so that $\mathbf{E}+i \mathbf{F}$ is a rank 1 complex matrix. Clearly, the proposition's cases 8, 9, and 12 span complex pencils which fall into the theorem's case (1). The proposition's cases $7^{\prime}$ and $9^{\prime}$ correspond to the theorem's cases (2) and (3), respectively. The only remaining rank 1 combination $\mathbf{E}+i \mathbf{F}$ in the proposition is case $5 c$, with $\zeta=0$ and $\epsilon=1$, and a calculation shows this corresponds to the theorem's case (6) with $t=1$. More calculations will check that these rank 1 pencils indeed form four different real equivalence classes.

The second main part of the proof is to sort the remaining fourteen rank 2 cases of the proposition into real equivalence classes of complex subspaces.

$$
\text { Let } \mathbf{K}_{1}=\left[\begin{array}{ccc}
i & 1 & 0 \\
0 & i & 1
\end{array}\right] \text {, from the generic case } 1 \text { of the proposition, and case (8) }
$$

of the theorem. For each $\gamma \in \mathbb{R}$, let $\mathbf{K}_{2}(\gamma)=\left[\begin{array}{ccc}i & 1 & 0 \\ 0 & 0 & 1+i \gamma\end{array}\right]$, in cases $1^{\prime}$ and 6 of the proposition.

Rather than going through all the matrix calculations, some of which are not much more than verification of uniqueness claims from Proposition 5.3, we will just briefly work out one case and even more briefly sketch the rest, leaving the details to the reader. The following calculation will show that the complex pencil spanned by $\mathbf{K}_{1}$ is not in the same real equivalence class as any pencil spanned by a matrix with 0 entries in the $(2,1)$ and $(2,2)$ positions. Suppose there were real invertible matrices $\mathbf{L}=\left[\begin{array}{cc}\ell_{1,1} & \ell_{1,2} \\ \ell_{2,1} & \ell_{2,2}\end{array}\right]$, and $\mathbf{R}_{3 \times 3}$, with nonzero columns $\left(\mathbf{r}^{1}, \mathbf{r}^{2}, \mathbf{r}^{3}\right)$, and a nonzero $\lambda \in \mathbb{C}$ 
such that

$$
\left[\begin{array}{ccc}
k_{1,1} & k_{1,2} & k_{1,3} \\
0 & 0 & k_{2,3}
\end{array}\right]=\lambda \mathbf{L}\left[\begin{array}{ccc}
i & 1 & 0 \\
0 & i & 1
\end{array}\right] \mathbf{R}
$$

Comparing the left two columns implies

$$
\lambda^{-1}\left[\begin{array}{cc}
k_{1,1} & k_{1,2} \\
0 & 0
\end{array}\right]=\mathbf{L}\left[\begin{array}{ccc}
i & 1 & 0 \\
0 & i & 1
\end{array}\right]\left(\mathbf{r}^{1}, \mathbf{r}^{2}\right)_{3 \times 2},
$$

and considering the real and imaginary parts of the lower row gives

$$
\left[\begin{array}{ll}
0 & 0
\end{array}\right]=\left(\ell_{2,1}, \ell_{2,2}\right)\left[\begin{array}{lll}
0 & 1 & 0 \\
0 & 0 & 1
\end{array}\right]\left(\mathbf{r}^{1}, \mathbf{r}^{2}\right)=\left(\ell_{2,1}, \ell_{2,2}\right)\left[\begin{array}{lll}
1 & 0 & 0 \\
0 & 1 & 0
\end{array}\right]\left(\mathbf{r}^{1}, \mathbf{r}^{2}\right)
$$

Since there are linearly independent vectors $\left(0, \ell_{2,1}, \ell_{2,2}\right)$ and $\left(\ell_{2,1}, \ell_{2,2}, 0\right)$ in the left kernel of $\left(\mathbf{r}^{1}, \mathbf{r}^{2}\right)$, it has rank 1 , which contradicts the requirement that $\mathbf{R}$ is invertible. The conclusion is that the complex pencil spanned by $\mathbf{K}_{1}$ is not in the same real equivalence class as the complex pencil spanned by $\mathbf{K}_{2}(\gamma)$, for any $\gamma$.

Another calculation, left to the reader, will show that the pencil spanned by $\mathbf{K}_{1}$ is not in the same real equivalence class as any pencil spanned by a matrix with a zero column, and similarly, for any $\gamma \in \mathbb{R}$, neither is the pencil spanned by $\mathbf{K}_{2}(\gamma)$. The pencil spanned by $\left[\begin{array}{ccc}i & 0 & 0 \\ 0 & i & 1\end{array}\right]$ in case 2 of the proposition is in the same real equivalence class as the pencil spanned by $\mathbf{K}_{2}(0)$. In fact, all the matrices $\mathbf{K}_{2}(\gamma)$ are equivalent to a nonzero complex scalar multiple of $\mathbf{K}_{2}(0)$ :

$$
\left[\begin{array}{ccc}
i & 1 & 0 \\
0 & 0 & 1+i \gamma
\end{array}\right]\left[\begin{array}{ccc}
1 & \gamma & 0 \\
-\gamma & 1 & 0 \\
0 & 0 & 1
\end{array}\right]=(1+i \gamma)\left[\begin{array}{ccc}
i & 1 & 0 \\
0 & 0 & 1
\end{array}\right]
$$

and this gives case (7) of the theorem.

The rest of the cases of the proposition, $3,4,5 a, 5 b, 5 c, 7,10,11,10^{\prime}, 4^{\prime}$, all give $\mathbf{E}+i \mathbf{F}$ with rank 2 and a zero first column, so they do not fall in the previously covered cases (1), (2), (3), (7), or (8) of the theorem, nor in case (6) with $t=1$. By Theorem 4.1, if $\mathbf{E}+i \mathbf{F}$ is a complex matrix with rank 2 and a zero first column, then there exist $2 \times 2$ invertible real matrices $\mathbf{A}_{3}$ and $\mathbf{A}_{4}$ and a nonzero $\lambda \in \mathbb{C}$ so that

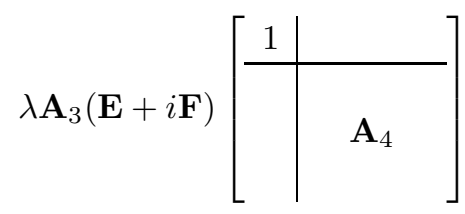

equals one of the matrices in this theorem's cases (4), (5) and (6). It remains only to check the claim that those classes, with their invariants $\theta$ and $t$, are still distinct. It 
is easily checked that there exist $\mathbf{A}_{5} \in G L(2, \mathbb{R}), \mathbf{A}_{6} \in G L(3, \mathbb{R})$ and a nonzero $\lambda \in \mathbb{C}$ such that

$$
\lambda \mathbf{A}_{5}\left[\begin{array}{lll}
0 & e & f \\
0 & g & h
\end{array}\right] \mathbf{A}_{6}=\left[\begin{array}{lll}
0 & p & q \\
0 & r & s
\end{array}\right]
$$

if and only if there exist $\mathbf{A}_{7}, \mathbf{A}_{8} \in G L(2, \mathbb{R})$ and a nonzero $\mu \in \mathbb{C}$ such that

$$
\mu \mathbf{A}_{7}\left[\begin{array}{ll}
e & f \\
g & h
\end{array}\right] \mathbf{A}_{8}=\left[\begin{array}{ll}
p & q \\
r & s
\end{array}\right] .
$$

For this, one can choose $\lambda=\mu, \mathbf{A}_{5}=\mathbf{A}_{7}$, and $\mathbf{A}_{8}$ equal to the lower right $2 \times 2$ block of $\mathbf{A}_{6}$. The claim now follows from Theorem 4.1. $\mathbf{}$

Although not necessary for the above proof, it is easy to check that cases $3,5 a$, $7,11,10^{\prime}, 4^{\prime}$ of the proposition fall into case (5) of Theorem 4.1, cases $4,5 b, 10$ of the proposition fall into case (4) of the theorem, and the rank 2 case of $5 c$ corresponds to the rank 2 case of (6).

In the following list of examples, we will consider the various geometric properties of maps of the form $Q \circ s \circ(\delta \times \delta)$, for each of the $r$-equivalence classes of matrices $\mathbf{Q}$, corresponding to the normal forms in the previous theorem.

The two cases where the kernel is spanned by a real matrix ((1), and (5) with $\theta=0$ ) represent projections where the center of projection is in $\delta_{5}\left(\mathbb{R} P^{5}\right) \subseteq \mathbb{C} P^{5}$, and so the image of $Q \circ s \circ(\delta \times \delta)$ is contained in some real projective 4-space, $\left(Q \circ \delta_{5}\right)\left(\mathbb{R} P^{5}\right)=\delta_{4}\left(\mathbb{R} P^{4}\right) \subseteq \mathbb{C} P^{4}$.

In the remaining cases, the center of projection is outside $\delta_{5}\left(\mathbb{R} P^{5}\right)$, and the image of $Q \circ s \circ(\delta \times \delta)$ is contained in $\left(Q \circ \delta_{5}\right)\left(\mathbb{R} P^{5}\right) \subseteq \mathbb{C} P^{4}$. In each of the examples, it will be easy to pick at least one complex affine neighborhood $U \subseteq \mathbb{C} P^{4}$ such that $U \cap\left(\left(Q \circ \delta_{5}\right)\left(\mathbb{R} P^{5}\right)\right)$ is a real affine 5 -space in $U$, but this won't help as much with the visualization as it did in the previous section. While $Q \circ \delta_{5}: \mathbb{R} P^{5} \rightarrow \mathbb{C} P^{4}$ is one-to-one on some affine neighborhoods, it is not an immersion of $\mathbb{R} P^{5}$, since in each case there is at least one real line which is collapsed to a point.

It will also be useful to observe how complex projective lines in $\mathbb{C} P^{4}$ meet the three-dimensional image of $Q \circ s \circ(\delta \times \delta)$, and the set $\left(Q \circ \delta_{5}\right)\left(\mathbb{R} P^{5}\right)$ which contains the real threefold. In a complex affine neighborhood $U$ whose intersection with $(Q \circ$ $\left.\delta_{5}\right)\left(\mathbb{R} P^{5}\right)$ is a real affine 5 -space, that 5 -space will contain a parallel family of complex affine lines, and every other complex line in $U$ will either be disjoint from the 5space, or will meet it in a point or a real affine line. In some other complex affine neighborhood $V$, those complex lines still look like complex lines, but $V \cap((Q \circ$ $\left.\left.\delta_{5}\right)\left(\mathbb{R} P^{5}\right)\right)$ may not be a real affine space. It contains a family of complex lines, but the previously mentioned real affine lines inside complex lines may transform from lines to circles or lines. 
It will be convenient to label some of the affine neighborhoods in the domain and the target:

$$
\begin{aligned}
& U_{00}=\left\{\left(\left[1: u_{1}: u_{2}\right],\left[1: v_{1}\right]\right)\right\}, \\
& U_{10}=\left\{\left(\left[u_{0}: 1: u_{2}\right],\left[1: v_{1}\right]\right)\right\}, \\
& U_{20}=\left\{\left(\left[u_{0}: u_{1}: 1\right],\left[1: v_{1}\right]\right)\right\}, \\
& U_{01}=\left\{\left(\left[1: u_{1}: u_{2}\right],\left[v_{0}: 1\right]\right)\right\}, \\
& U_{11}=\left\{\left(\left[u_{0}: 1: u_{2}\right],\left[v_{0}: 1\right]\right)\right\}, \\
& U_{21}=\left\{\left(\left[u_{0}: u_{1}: 1\right],\left[v_{0}: 1\right]\right)\right\} \subseteq \mathbb{R} P^{2} \times \mathbb{R} P^{1}, \\
& V_{0}=\left\{\left[1: Z_{1}: Z_{2}: Z_{3}: Z_{4}\right]\right\}, \\
& \vdots \quad \vdots \\
& V_{4}=\left\{\left[Z_{0}: Z_{1}: Z_{2}: Z_{3}: 1\right]\right\} \subseteq \mathbb{C} P^{4} .
\end{aligned}
$$

EXAMPLE 5.5. The rank 1 matrix in case (1) of Theorem 5.4 has already appeared in Example 5.1; it corresponds to the center of projection being an element of the real submanifold $(s \circ(\delta \times \delta))\left(\mathbb{R} P^{2} \times \mathbb{R} P^{1}\right)$. Using the matrix $\mathbf{Q}$ from that example, the map

$$
\begin{aligned}
Q \circ s \circ(\delta \times \delta): \mathbb{R} P^{2} \times \mathbb{R} P^{1} & \rightarrow \mathbb{C} P^{4}: \\
\left(\left[u_{0}: u_{1}: u_{2}\right],\left[v_{0}: v_{1}\right]\right) & \mapsto\left[u_{0} v_{1}: u_{1} v_{0}: u_{1} v_{1}: u_{2} v_{0}: u_{2} v_{1}\right]
\end{aligned}
$$

has geometric properties analogous to those of $Q \circ s$. It is undefined at one point of the domain: the base point of $Q \circ s$ is an element of the image of $\delta \times \delta$. The image of $Q \circ s \circ(\delta \times \delta)$ is contained in $\delta_{4}\left(\mathbb{R} P^{4}\right) \subseteq \mathbb{C} P^{4}$, so $Q \circ s \circ(\delta \times \delta)$ is a smooth but singular map from a punctured $\mathbb{R} P^{2} \times \mathbb{R} P^{1}$ to a real projective 4 -space.

EXAMPLE 5.6. Another rank 1 matrix in Theorem 5.4 is case (2), where a representative coefficient matrix, i.e., one with a kernel spanned by vec $\left(\left[\begin{array}{lll}0 & 1 & i \\ 0 & 0 & 0\end{array}\right]\right)$, is

$$
\mathbf{Q}=\left[\begin{array}{llllll}
1 & 0 & 0 & 0 & 0 & 0 \\
0 & 1 & 0 & 0 & 0 & 0 \\
0 & 0 & 1 & 0 & i & 0 \\
0 & 0 & 0 & 1 & 0 & 0 \\
0 & 0 & 0 & 0 & 0 & 1
\end{array}\right]
$$

The induced map is

$$
Q \circ s \circ(\delta \times \delta):\left(\left[u_{0}: u_{1}: u_{2}\right],\left[v_{0}: v_{1}\right]\right) \mapsto\left[u_{0} v_{0}: u_{0} v_{1}: u_{1} v_{0}+i u_{2} v_{0}: u_{1} v_{1}: u_{2} v_{1}\right]
$$


which is defined on all of $\mathbb{R} P^{2} \times \mathbb{R} P^{1}$, but $Q \circ s$ has a base point at ([0:1:i], [1: $0]) \in \mathbb{C} P^{2} \times \mathbb{C} P^{1}$. The singular locus of $Q \circ s$ is, as in Example 5.1,

$$
\Sigma_{3}=\left\{z_{0} w_{1}=\left(z_{1}+i z_{2}\right) w_{1}=0\right\} \backslash\{([0: 1: i],[1: 0])\} .
$$

The real projective plane $\left\{\left(\left[u_{0}: u_{1}: u_{2}\right],[1: 0]\right)\right\}$ is the intersection of the image of $\delta \times \delta$ with $\Sigma_{3}$. The restriction of $Q \circ s \circ(\delta \times \delta)$ maps this plane onto a complex projective line $\mathcal{T}: Q \circ s \circ(\delta \times \delta):\left(\left[u_{0}: u_{1}: u_{2}\right],[1: 0]\right) \mapsto\left[u_{0}: 0: u_{1}+i u_{2}: 0: 0\right]$, and it is one-to-one except that the line $\left\{u_{0}=0\right\}$ is mapped to the point $[0: 0: 1: 0: 0]$. Away from this plane, $Q \circ s \circ(\delta \times \delta)$ is a totally real immersion.

To get a complete picture of the local geometric properties of the image of $Q \circ s \circ$ $(\delta \times \delta)$, we can consider its restrictions to the six affine neighborhoods whose union covers the domain.

$Q \circ s \circ(\delta \times \delta)$ restricts to the $U_{21} \rightarrow V_{4}$ neighborhoods, with the image contained in a real 5 -subspace of $V_{4}$ :

$$
\left(u_{0}, u_{1}, v_{0}\right) \mapsto\left(X_{0}, X_{1}, X_{2}, Y_{2}, X_{3}\right)=\left(u_{0} v_{0}, u_{0}, u_{1} v_{0}, v_{0}, u_{1}\right) .
$$

The image is a totally real polynomial graph over the $X_{1}, Y_{2}, X_{3}$ subspace. All of the complex lines contained in this real 5-space are of the form $\left\{\left[r_{0}: r_{1}: Z_{2}: r_{3}: 1\right]: Z_{2} \in\right.$ $\mathbb{C}\}$ for some real $r_{0}, r_{1}, r_{3}$. Each complex line of the form $\left\{\left[0: 0: Z_{2}: r_{3}: 1\right]\right\}$ meets the threefold along a real line $\left\{\left(X_{0}, X_{1}, X_{2}, Y_{2}, X_{3}\right)=\left(0,0, r_{3} v_{0}, v_{0}, r_{3}\right): v_{0} \in \mathbb{R}\right\}$, and each of the other complex lines in this 5-space meets it in no more than one point. The restriction to the $U_{11} \rightarrow V_{3}$ neighborhoods is similar.

The restriction of $Q \circ s \circ(\delta \times \delta)$ to a parametric map $U_{01} \rightarrow V_{1}$ :

$$
\left(u_{1}, u_{2}, v_{0}\right) \mapsto\left(X_{0}, X_{2}, Y_{2}, X_{3}, X_{4}\right)=\left(v_{0}, u_{1} v_{0}, u_{2} v_{0}, u_{1}, u_{2}\right),
$$

gives a totally real polynomial graph over the $X_{0}, X_{3}, X_{4}$ subspace. Each complex line in this 5 -space meets the threefold at exactly one point.

Restricting to another affine neighborhood shows part of the image of the singular locus. Consider the parametric map $Q \circ s \circ(\delta \times \delta): U_{00} \rightarrow V_{0}$ :

$$
\left(u_{1}, u_{2}, v_{1}\right) \mapsto\left(X_{1}, X_{2}, Y_{2}, X_{3}, X_{4}\right)=\left(v_{1}, u_{1}, u_{2}, u_{1} v_{1}, u_{2} v_{1}\right) .
$$

Its image is a smoothly embedded graph over the $X_{1}, X_{2}, Y_{2}$ subspace, and the graph is tangent at the origin to this subspace. The image of the real plane $\left\{v_{1}=0\right\}$ is the complex line $\mathcal{T} \cap V_{0}=$ the $Z_{2}$-axis in $V_{0}$, which is the CR singular locus of the image of $Q \circ s \circ(\delta \times \delta)$. Each of the other complex lines in this 5 -space meets the threefold at no more than one point. 
In the $\left(Z_{1}, Z_{2}, Z_{3}, Z_{4}\right)$ coordinates of $V_{0}$, the implicit equations for the image are

$$
\left\{Y_{1}=0, Z_{3}=\frac{1}{2}\left(Z_{2}+\bar{Z}_{2}\right) X_{1}, Z_{4}=\frac{1}{2 i}\left(Z_{2}-\bar{Z}_{2}\right) X_{1}\right\}
$$

The point at the origin is then seen to be a CR singularity of type (XI) from the classification of [7], and in fact each of the other points in the CR singular locus is also of type (XI).

Restricting to the $U_{20} \rightarrow V_{2}$ neighborhoods shows the differential-topological singularity:

$$
\begin{aligned}
Q \circ s \circ(\delta \times \delta):\left(\left[u_{0}: u_{1}: 1\right],\left[1: v_{1}\right]\right) & \mapsto\left[u_{0}: u_{0} v_{1}: u_{1}+i: u_{1} v_{1}: v_{1}\right] \\
& =\left[\frac{u_{0}}{u_{1}+i}: \frac{u_{0} v_{1}}{u_{1}+i}: 1: \frac{u_{1} v_{1}}{u_{1}+i}: \frac{v_{1}}{u_{1}+i}\right] .
\end{aligned}
$$

This restriction can be written as a real cubic rational parametrization

$$
\begin{aligned}
\left(u_{0}, u_{1}, v_{1}\right) & \mapsto\left(X_{0}, Y_{0}, X_{1}, Y_{1}, X_{3}, Y_{3}, X_{4}, Y_{4}\right) \\
& =\left(\frac{u_{0} u_{1}}{u_{1}^{2}+1}, \frac{-u_{0}}{u_{1}^{2}+1}, \frac{u_{0} u_{1} v_{1}}{u_{1}^{2}+1}, \frac{-u_{0} v_{1}}{u_{1}^{2}+1}, \frac{u_{1}^{2} v_{1}}{u_{1}^{2}+1}, \frac{-u_{1} v_{1}}{u_{1}^{2}+1}, \frac{u_{1} v_{1}}{u_{1}^{2}+1}, \frac{-v_{1}}{u_{1}^{2}+1}\right) .
\end{aligned}
$$

The Jacobian of this parametric map has rank 3, except along the line $\left\{u_{0}=v_{1}=0\right\}$, where it has rank 2, and the map takes all the points on the line to the origin of $V_{2}$. The plane $\left\{v_{1}=0\right\}$ in the domain is mapped to the $Z_{0}$-axis of $V_{2}$, but the points on the real $X_{0}$-axis, except for the origin, are not in the image of $U_{20}$. All the points in the image of $U_{20}$ in $V_{2}$ satisfy the implicit equations:

$$
\begin{gathered}
0=Y_{1}+X_{0} X_{4}+Y_{0} Y_{4}, \quad 0=X_{1}-X_{0} X_{3}+X_{0} Y_{4}, \quad 0=X_{4}^{2}+X_{3} Y_{4}, \\
0=Y_{1} X_{4}-X_{1} Y_{4}, \quad 0=Y_{1} X_{3}+X_{1} X_{4}, \quad 0=Y_{0} X_{4}-X_{0} Y_{4}, \\
0=Y_{0} X_{3}+X_{0} X_{4}, \quad 0=Y_{0} X_{1}-X_{0} Y_{1}, \quad 0=Y_{3}+X_{4} .
\end{gathered}
$$

More than five equations are required to get the smallest three-dimensional real affine variety in $V_{2}$ containing the image of $U_{20}$, but the variety still contains some points not in the image of $U_{20}$; specifically, the points on the $X_{0}$-axis satisfy all the above equations. In fact, all of the $Z_{0}$-axis is contained in the image of $Q \circ s \circ(\delta \times \delta)$, since the non-zero points on the $X_{0}$-axis are in the previously considered image of $U_{00}$. A computation checks that the singular locus of the above real variety contains only the origin. The image of $U_{10} \rightarrow V_{2}$ is similar.

EXAMPLE 5.7. The rank 1 matrix in case (3) of Theorem 5.4 has a representative 
coefficient matrix

$$
\mathbf{Q}=\left[\begin{array}{llllll}
1 & 0 & 0 & 0 & 0 & 0 \\
0 & 1 & 0 & 0 & 0 & 0 \\
0 & 0 & 1 & i & 0 & 0 \\
0 & 0 & 0 & 0 & 1 & 0 \\
0 & 0 & 0 & 0 & 0 & 1
\end{array}\right]
$$

and the induced map is

$$
Q \circ s \circ(\delta \times \delta):\left(\left[u_{0}: u_{1}: u_{2}\right],\left[v_{0}: v_{1}\right]\right) \mapsto\left[u_{0} v_{0}: u_{0} v_{1}: u_{1} v_{0}+i u_{1} v_{1}: u_{2} v_{0}: u_{2} v_{1}\right]
$$

which is defined on all of $\mathbb{R} P^{2} \times \mathbb{R} P^{1}$, but $Q \circ s$ has a base point at $([0: 1: 0],[1$ : $i]) \in \mathbb{C} P^{2} \times \mathbb{C} P^{1}$. The singular locus of $Q \circ s$ is, as in Example 5.1,

$$
\Sigma_{3}=\left\{z_{0}\left(w_{0}+i w_{1}\right)=z_{2}\left(w_{0}+i w_{1}\right)=0\right\} \backslash\{([0: 1: 0],[1: i])\}
$$

The real projective line $\left\{\left([0: 1: 0],\left[v_{0}: v_{1}\right]\right)\right\}$ is the intersection of the image of $\delta \times \delta$ with $\Sigma_{3} . Q \circ s \circ(\delta \times \delta)$ maps this line onto the point $\mathcal{X}=[0: 0: 1: 0: 0]$, and away from this line, $Q \circ s \circ(\delta \times \delta)$ is a totally real immersion.

$$
Q \circ s \circ(\delta \times \delta) \text { restricts to a parametric map } U_{21} \rightarrow V_{4}:
$$

$$
\left(u_{0}, u_{1}, v_{0}\right) \mapsto\left(X_{0}, X_{1}, X_{2}, Y_{2}, X_{3}\right)=\left(u_{0} v_{0}, u_{0}, u_{1} v_{0}, u_{1}, v_{0}\right)
$$

The image is a totally real polynomial graph over the $X_{1}, Y_{2}, X_{3}$ subspace. All of the complex lines contained in this real 5 -space are of the form $\left\{\left[r_{0}: r_{1}: Z_{2}: r_{3}: 1\right]: Z_{2} \in\right.$ $\mathbb{C}\}$ for some real $r_{0}, r_{1}, r_{3}$. Each complex line of the form $\left\{\left[r_{1} r_{3}: r_{1}: Z_{2}: r_{3}: 1\right]\right\}$ meets the threefold along a real line $\left\{\left(X_{0}, X_{1}, X_{2}, Y_{2}, X_{3}\right)=\left(r_{1} r_{3}, r_{1}, r_{3} u_{1}, u_{1}, r_{3}\right)\right.$ : $\left.u_{1} \in \mathbb{R}\right\}$, and each of the other complex lines in this 5 -space is disjoint from it. The restrictions to the $U_{01} \rightarrow V_{1}, U_{00} \rightarrow V_{0}$ and $U_{20} \rightarrow V_{3}$ neighborhoods are similar, and the $V_{0}, V_{1}, V_{3}, V_{4}$ neighborhoods cover $\mathbb{C} P^{4}$ except for one point.

Restricting to $U_{11} \rightarrow V_{2}$ shows the differential-topological singularity:

$$
\begin{aligned}
Q \circ s \circ(\delta \times \delta):\left(\left[u_{0}: 1: u_{2}\right],\left[v_{0}: 1\right]\right) & \mapsto\left[u_{0} v_{0}: u_{0}: v_{0}+i: u_{2} v_{0}: u_{2}\right] \\
& =\left[\frac{u_{0} v_{0}}{v_{0}+i}: \frac{u_{0}}{v_{0}+i}: 1: \frac{u_{2} v_{0}}{v_{0}+i}: \frac{u_{2}}{v_{0}+i}\right],
\end{aligned}
$$

and it can be written as a real cubic rational parametrization

$$
\begin{aligned}
\left(u_{0}, u_{2}, v_{0}\right) & \mapsto\left(X_{0}, Y_{0}, X_{1}, Y_{1}, X_{3}, Y_{3}, X_{4}, Y_{4}\right) \\
& =\left(\frac{u_{0} v_{0}^{2}}{v_{0}^{2}+1}, \frac{-u_{0} v_{0}}{v_{0}^{2}+1}, \frac{u_{0} v_{0}}{v_{0}^{2}+1}, \frac{-u_{0}}{v_{0}^{2}+1}, \frac{u_{2} v_{0}^{2}}{v_{0}^{2}+1}, \frac{-u_{2} v_{0}}{v_{0}^{2}+1}, \frac{u_{2} v_{0}}{v_{0}^{2}+1}, \frac{-u_{2}}{v_{0}^{2}+1}\right) .
\end{aligned}
$$


The Jacobian of this parametric map has rank 3, except along the line $\left\{u_{0}=u_{2}=0\right\}$, where it has rank 2, and the map takes all the points on the line to the point $\mathcal{X}=$ the origin of $V_{2}$. The image of $U_{11}$ in $V_{2}$ satisfies the homogeneous implicit equations:

$$
\begin{gathered}
0=X_{0} Y_{1}+X_{1}^{2}, \quad 0=X_{3} Y_{4}+X_{4}^{2}, \quad 0=X_{0} X_{4}-X_{1} X_{3}, \quad 0=X_{0} Y_{4}-X_{3} Y_{1}, \\
0=X_{1} X_{4}+X_{3} Y_{1}, \quad 0=X_{4} Y_{1}-X_{1} Y_{4}, \quad 0=X_{1}+Y_{0}, \quad 0=X_{4}+Y_{3} .
\end{gathered}
$$

A computation verifies that this is a three-dimensional real affine variety with a singularity only at the origin. The image of $U_{10} \rightarrow V_{2}$ is similar.

EXAMPLE 5.8. The rank 2 matrix, $\left[\begin{array}{ccc}0 & 0 & 1 / 2 \\ 0 & 1 / 2 & i\end{array}\right]$ in case (4) of Theorem 5.4, has a representative coefficient matrix

$$
\mathbf{Q}=\left[\begin{array}{cccccc}
1 & 0 & 0 & 0 & 0 & 0 \\
0 & 1 & 0 & 0 & 0 & 0 \\
0 & 0 & 1 & 0 & 0 & 0 \\
0 & 0 & 0 & 1 & -1 & 0 \\
0 & 0 & 0 & 0 & 2 & i
\end{array}\right]
$$

The induced map $Q \circ s \circ(\delta \times \delta)$ is given by the formula

$$
\left(\left[u_{0}: u_{1}: u_{2}\right],\left[v_{0}: v_{1}\right]\right) \mapsto\left[u_{0} v_{0}: u_{0} v_{1}: u_{1} v_{0}: u_{1} v_{1}-u_{2} v_{0}: 2 u_{2} v_{0}+i u_{2} v_{1}\right] .
$$

The singular locus of $Q \circ s$ is, as in Example 5.2:

$$
\Sigma_{4}=\left\{z_{0}=2 z_{1} w_{0}+i\left(z_{1} w_{1}+z_{2} w_{0}\right)=0\right\},
$$

and its two-to-one locus $\Theta$ is given by $\left\{z_{0}=0\right\}$, so that the restriction of $Q \circ s$ to $\Theta$ is exactly as in Example 4.5, and we can conclude that $Q \circ s \circ(\delta \times \delta)$ is one-to-one. One real line in $\delta_{5}\left(\mathbb{R} P^{5}\right)$ is projected by $Q$ to a point: $Q:\left\{\left[0: 0: 0: u_{3}: u_{3}:\right.\right.$ $\left.\left.u_{5}\right]\right\} \mapsto[0: 0: 0: 0: 1]$, and this line meets the image of $s \circ(\delta \times \delta)$ exactly once, at $(s \circ(\delta \times \delta))(([0: 0: 1],[0: 1]))=[0: 0: 0: 0: 0: 1]$.

The image of $\delta \times \delta$ in $\mathbb{C} P^{2} \times \mathbb{C} P^{1}$ intersects $\Sigma_{4}$ in exactly one point, ([0:0: $1]$, $[0: 1])$, so we can conclude that on the complement of that point, $Q \circ s \circ(\delta \times \delta)$ is a totally real immersion. It remains only to check the behavior of $Q \circ s \circ(\delta \times \delta)$ at the point $([0: 0: 1],[0: 1])$, and in fact it has a singularity there as a smooth map, where the rank of the (real) Jacobian drops to 2 at that point.

$$
\begin{aligned}
& Q \circ s \circ(\delta \times \delta) \text { restricts to a parametric map } U_{10} \rightarrow V_{2}: \\
& \quad\left(u_{0}, u_{2}, v_{1}\right) \mapsto\left(X_{0}, X_{1}, X_{3}, X_{4}, Y_{4}\right)=\left(u_{0}, u_{0} v_{1}, v_{1}-u_{2}, 2 u_{2}, u_{2} v_{1}\right) .
\end{aligned}
$$

The image is a totally real polynomial graph over the $X_{0}, X_{3}, X_{4}$ subspace. All of the complex lines contained in this real 5 -space are of the form $\left\{\left[r_{0}: r_{1}: 1: r_{3}: Z_{4}\right]: Z_{4} \in\right.$ 
$\mathbb{C}\}$ for some real $r_{0}, r_{1}, r_{3}$. Each complex line of the form $\left\{\left[0: 0: 1: r_{3}: Z_{4}\right]\right\}$ meets the threefold along the parabola $\left\{\left(X_{0}, X_{1}, X_{3}, X_{4}, Y_{4}\right)=\left(0,0, r_{3}, 2 u_{2}, u_{2}\left(r_{3}+u_{2}\right)\right)\right.$ : $\left.u_{2} \in \mathbb{R}\right\}$, and each of the other complex lines in this 5 -space meets it at no more than one point. The occurrence of these parabolas is enough to distinguish this image from images in any other r-equivalence class. The restrictions to the $U_{01} \rightarrow V_{1}$ and $U_{00} \rightarrow V_{0}$ neighborhoods are also totally real graphs, but do not show the above parabolic intersections.

The singularity of the image of $Q \circ s \circ(\delta \times \delta)$ is not in the image of the $U_{20}$ or $U_{11}$ neighborhoods, so we consider the neighborhoods $U_{21} \rightarrow V_{4}$.

$$
\begin{aligned}
Q \circ s \circ(\delta \times \delta):\left(\left[u_{0}: u_{1}: 1\right],\left[v_{0}: 1\right]\right) & \mapsto\left[u_{0} v_{0}: u_{0}: u_{1} v_{0}: u_{1}-v_{0}: 2 v_{0}+i\right] \\
& =\left[\frac{u_{0} v_{0}}{2 v_{0}+i}: \frac{u_{0}}{2 v_{0}+i}: \frac{u_{1} v_{0}}{2 v_{0}+i}: \frac{u_{1}-v_{0}}{2 v_{0}+i}: 1\right],
\end{aligned}
$$

and it can be written as a real cubic rational parametrization

$$
\begin{gathered}
\left(u_{0}, u_{2}, v_{0}\right) \mapsto\left(X_{0}, Y_{0}, X_{1}, Y_{1}, X_{2}, Y_{2}, X_{3}, Y_{3}\right) \\
=\left(\frac{2 u_{0} v_{0}^{2}}{4 v_{0}^{2}+1}, \frac{-u_{0} v_{0}}{4 v_{0}^{2}+1}, \frac{2 u_{0} v_{0}}{4 v_{0}^{2}+1}, \frac{-u_{0}}{4 v_{0}^{2}+1}, \frac{2 u_{1} v_{0}^{2}}{4 v_{0}^{2}+1}, \frac{-u_{1} v_{0}}{4 v_{0}^{2}+1}, \frac{2\left(u_{1}-v_{0}\right) v_{0}}{4 v_{0}^{2}+1}, \frac{v_{0}-u_{1}}{4 v_{0}^{2}+1}\right) .
\end{gathered}
$$

The Jacobian of this parametric map has rank 3, except at the point $\left(u_{0}, u_{1}, v_{0}\right)=$ $(0,0,0)$, where it has rank 2 . The image of $U_{21}$ in $V_{4}$ satisfies the equations:

$$
\begin{aligned}
& 0=X_{3}^{2}+2 X_{3}^{3}+4 X_{2} X_{3} Y_{3}+2 X_{3} Y_{3}^{2}+4 Y_{2} Y_{3}^{2}, \\
& 0=X_{2} X_{3}+2 X_{2} X_{3}^{2}+4 X_{2}^{2} Y_{3}+4 Y_{2}^{2} Y_{3}+2 X_{2} Y_{3}^{2}, \\
& 0=X_{2}^{2}+2 X_{2}^{2} X_{3}+4 X_{2}^{2} Y_{2}+4 Y_{2}^{3}+2 X_{2} Y_{2} Y_{3}, \\
& 0=X_{0}+2 X_{0} X_{3}+4 X_{0} Y_{2}-2 Y_{1} Y_{2}+2 Y_{0} Y_{3}, \\
& 0=2 Y_{0}^{2}+X_{0} Y_{1}, \quad 0=X_{3} Y_{2}-X_{2} Y_{3}, \\
& 0=X_{3} Y_{1}+2 Y_{0} Y_{3}, \quad 0=X_{3} Y_{0}-X_{0} Y_{3}, \\
& 0=X_{2} Y_{1}+2 Y_{0} Y_{2}, \quad 0=X_{2} Y_{0}-X_{0} Y_{2}, \\
& 0=X_{1}+2 Y_{0} .
\end{aligned}
$$

A computation verifies that this is a three-dimensional real affine variety with a singularity only at the origin.

ExAmple 5.9. The matrix in case (5), with $\alpha=1$, has a real representative coefficient matrix, which has already appeared in Example 5.2. The map $Q \circ s \circ(\delta \times \delta)$ is defined on all of $\mathbb{R} P^{2} \times \mathbb{R} P^{1}$, and is singular along a real curve. A subset of the domain, $\left\{u_{0}=0\right\}$ (the real analogue of $\Theta$ in Example 5.2), is a two-to-one locus. The restriction of the map to this locus is exactly as in Example 4.6. The image 
of $Q \circ s \circ(\delta \times \delta)$ is contained in $\delta_{4}\left(\mathbb{R} P^{4}\right) \subseteq \mathbb{C} P^{4}$, so it can be considered as a real projection of the real Segre variety to a real projective 4 -space. Taking coordinates $\left[X_{0}: \cdots: X_{4}\right]$ for $\mathbb{R} P^{4}$, the image of $Q \circ s \circ(\delta \times \delta)$ is contained in, but not equal to, the real projective hypersurface $\left\{X_{0} X_{1} X_{2}+X_{2}^{2} X_{4}-X_{1}^{2} X_{3}=0\right\}$. This real variety contains, as its singular locus, the real projective plane $\left\{X_{1}=X_{2}=0\right\}$. Any point in the intersection of the image of $Q \circ s \circ(\delta \times \delta)$ with that plane must be of the form $(Q \circ s \circ(\delta \times \delta))\left(\left(\left[0: u_{1}: u_{2}\right],\left[v_{1}: v_{2}\right]\right)\right)$, but as observed in Example 4.6 and Figure 4.4 , such points do not fill up the whole plane.

The map can also be considered as the $\theta \rightarrow 0^{+}$limiting case of the complex projections in the next example.

ExAmple 5.10. The remaining matrices in case (5), with $\alpha=e^{i \theta}, 0<\theta \leq \frac{\pi}{2}$, correspond to representative coefficient matrices of the form:

$$
\mathbf{Q}_{\alpha}=\left[\begin{array}{cccccc}
1 & 0 & 0 & 0 & 0 & 0 \\
0 & 1 & 0 & 0 & 0 & 0 \\
0 & 0 & \alpha & 0 & 0 & -1 \\
0 & 0 & 0 & 1 & 0 & 0 \\
0 & 0 & 0 & 0 & 1 & 0
\end{array}\right]
$$

For each $\alpha$, the induced map is

$$
Q_{\alpha} \circ s \circ(\delta \times \delta):\left(\left[u_{0}: u_{1}: u_{2}\right],\left[v_{0}: v_{1}\right]\right) \mapsto\left[u_{0} v_{0}: u_{0} v_{1}: \alpha u_{1} v_{0}-u_{2} v_{1}: u_{1} v_{1}: u_{2} v_{0}\right] \text {. }
$$

The singular locus of $Q_{\alpha} \circ s$ is, as in Example 5.2:

$$
\Sigma_{4}=\left\{z_{0}=\alpha z_{1} w_{0}+z_{2} w_{1}=0\right\},
$$

and its two-to-one locus $\Theta$ is given by $\left\{z_{0}=0\right\}$, so that the restriction of $Q_{\alpha} \circ s$ to $\Theta$ is exactly as in Example 4.7. We can conclude that $Q_{\alpha} \circ s \circ(\delta \times \delta)$ is one-to-one except for a double point,

$$
\begin{aligned}
{[0: 0: 1: 0: 0] } & =\left(Q_{\alpha} \circ s \circ(\delta \times \delta)\right)(([0: 0: 1],[0: 1])) \\
& =\left(Q_{\alpha} \circ s \circ(\delta \times \delta)\right)(([0: 1: 0],[1: 0])),
\end{aligned}
$$

and the image of $\delta \times \delta$ in $\mathbb{C} P^{1} \times \mathbb{C} P^{1}$ intersects $\Sigma_{4}$ in exactly two points, $([0: 1: 0],[0$ : $1])$ and $([0: 0: 1],[1: 0])$, so on the complement of these two points, $Q_{\alpha} \circ s \circ(\delta \times \delta)$ is a totally real immersion.

The image of the restriction of $Q_{\alpha} \circ s \circ(\delta \times \delta)$ to $U_{00} \rightarrow V_{0}$ is a totally real polynomial graph, and so is the image of $U_{01} \rightarrow V_{1}$. The restrictions to $U_{10} \rightarrow V_{2}$ and $U_{21} \rightarrow V_{2}$ are each one-to-one, but together they show the double point.

Restricting $Q_{\alpha} \circ s \circ(\delta \times \delta)$ to the $U_{11} \rightarrow V_{3}$ affine neighborhoods shows what's going on near one of the singular points of $Q \circ s$. Considering

$$
Q_{\alpha} \circ s \circ(\delta \times \delta):\left(\left[u_{0}: 1: u_{2}\right],\left[v_{0}: 1\right]\right) \mapsto\left[u_{0} v_{0}: u_{0}: \alpha v_{0}-u_{2}: 1: u_{2} v_{0}\right]
$$


as a map from $\mathbb{R}^{3}$ to the $X_{0}, X_{1}, X_{2}, Y_{2}, X_{4} 5$-space, this restriction is a smooth embedding, a polynomial graph over its tangent space at the origin, the $X_{1}, X_{2}, Y_{2}$ 3 -space which contains the $Z_{2}$-axis. This is the only $\mathrm{CR}$ singularity in this affine neighborhood, the submanifold being totally real at the other points. For $\alpha=a+i b$ with $b>0$, the image is given as a submanifold of $V_{3}$ by the equations

$$
\left\{Y_{1}=0, Z_{0}=\frac{1}{2 b i} X_{1}\left(Z_{2}-\bar{Z}_{2}\right), Z_{4}=\frac{1}{4 b^{2}}\left(2 a Z_{2} \bar{Z}_{2}-\bar{\alpha} Z_{2}^{2}-\alpha \bar{Z}_{2}^{2}\right)\right\} .
$$

The ratio of coefficients $\beta=\left|\frac{-\alpha}{2 a}\right| \in\left(\frac{1}{2}, \infty\right]$ resembles the Bishop invariant from Example 4.7, and in [7] it is shown that this quantity is a local biholomorphic invariant of the submanifold near the CR singularity. In the classification of [7], this CR singularity is of type (III) if $a>0$, and of type (VII) if $a=0$.

All of the complex lines contained in this 5-space are of the form $\left\{\left[r_{0}: r_{1}: Z_{2}\right.\right.$ : $\left.\left.1: r_{4}\right]: Z_{2} \in \mathbb{C}\right\}$ for some real $r_{0}, r_{1}, r_{4}$; one such line (with $r_{0}=r_{1}=r_{4}=0$ ) is the complex line contained in the tangent plane at the origin, and lines with $r_{0}=r_{1}=0$, $r_{4} \neq 0$ meet the submanifold in hyperbolas, as in Example 4.7.

The restriction to $U_{20} \rightarrow V_{4}$,

$$
Q_{\alpha} \circ s \circ(\delta \times \delta):\left(\left[u_{0}: u_{1}: 1\right],\left[1: v_{1}\right]\right) \mapsto\left[u_{0}: u_{0} v_{1}: \alpha u_{1}-v_{1}: u_{1} v_{1}: 1\right],
$$

is similar, showing the other CR singularity with the same numerical invariant. The CR singularities and the double point are both topologically unstable phenomena for immersions of real threefolds in a complex 4-manifold; small changes in the coefficient matrix will usually result in a totally real embedding.

EXAMPLE 5.11. The remaining rank 1 equivalence class in Theorem 5.4 is case (6), with $t=1$. A representative coefficient matrix is

$$
\mathbf{Q}_{1}=\left[\begin{array}{cccccc}
1 & 0 & 0 & 0 & 0 & 0 \\
0 & 1 & 0 & 0 & 0 & 0 \\
0 & 0 & 1 & 0 & 0 & 1 \\
0 & 0 & 0 & 1 & -1 & 0 \\
0 & 0 & 0 & 0 & 1 & i
\end{array}\right]
$$

The induced map $Q_{1} \circ s \circ(\delta \times \delta)$ is given by

$$
\left(\left[u_{0}: u_{1}: u_{2}\right],\left[v_{0}: v_{1}\right]\right) \mapsto\left[u_{0} v_{0}: u_{0} v_{1}: u_{1} v_{0}+u_{2} v_{1}: u_{1} v_{1}-u_{2} v_{0}: u_{2} v_{0}+i u_{2} v_{1}\right] .
$$

The composite $Q_{1} \circ s$ has its base point at $([0: 1: i],[1: i])$, and its singular locus is

$$
\left.\Sigma_{3}=\left\{z_{0}\left(w_{0}+i w_{1}\right)=\left(z_{1}+i z_{2}\right)\left(w_{0}+i w_{1}\right)=0\right\} \backslash\{[0: 1: i],[1: i])\right\} .
$$

The image of $\delta \times \delta$ in $\mathbb{C} P^{2} \times \mathbb{C} P^{1}$ is disjoint from $\Sigma_{3}$, and $Q_{1} \circ s \circ(\delta \times \delta)$ is defined at every point of $\mathbb{R} P^{2} \times \mathbb{R} P^{1}$, so we can conclude that $Q_{1} \circ s \circ(\delta \times \delta)$ is a totally real embedding. 
As in Example 4.8, this threefold has the property that if it meets a complex line in a curve, then that curve is a circle or line. To check this, we will consider a few pieces of the domain.

The restriction of $Q_{1} \circ s \circ(\delta \times \delta)$ to the $U_{00} \rightarrow V_{0}$ neighborhoods gives a real affine variety, parametrized by

$$
\left(u_{1}, u_{2}, v\right) \mapsto\left[1: v: u_{1}+u_{2} v: u_{1} v-u_{2}: u_{2}+i u_{2} v\right],
$$

and equal to the intersection of two real affine quadric hypersurfaces inside the $X_{1}, X_{2}$, $X_{3}, X_{4}, Y_{4} 5$-subspace. For a complex affine line in $V_{0}$, there are three possibilities. The complex line could meet this subspace in at most one point, which may or may not lie on the real threefold. The complex line could meet the 5 -subspace in a real affine line, which could meet the threefold in at most two points, or could be contained in it. Thirdly, the complex line could be contained in the 5 -subspace, where it has the form $\left\{\left[1: r_{1}: r_{2}: r_{3}: Z_{4}\right]: Z_{4} \in \mathbb{C}\right\}$, for some real $r_{1}, r_{2}, r_{3}$. Such a line meets the threefold if there is a real solution $\left(u_{1}, u_{2}, v\right)$ of the set of equations $v=r_{1}$, $u_{1}+u_{2} v=r_{2}, u_{1} v-u_{2}=r_{3}$, and it is easy to see that there is at most one such solution for any given $\left(r_{1}, r_{2}, r_{3}\right)$.

The next piece of the domain is the $\left\{u_{0}=0\right\}$ subset, where the parametric map restricts to

$$
\left(\left[0: u_{1}: u_{2}\right],\left[v_{1}, v_{2}\right]\right) \mapsto\left[0: 0: u_{1} v_{0}+u_{2} v_{1}: u_{1} v_{1}-u_{2} v_{0}: u_{2}\left(v_{0}+i v_{1}\right)\right] .
$$

The image of the restriction is contained in the complex projective plane $\mathcal{P}_{1}=\left\{Z_{0}=\right.$ $\left.Z_{1}=0\right\}$, and it is exactly as in Example 4.8. A complex projective line in $\mathbb{C} P^{4}$ can meet $\mathcal{P}_{1}$ in at most one point, or it can be contained in $\mathcal{P}_{1}$, in which case it can meet the surface in at most two points, or in a real line or circle.

The last piece of the domain is the $\left\{v_{0}=0\right\}$ subset, where the parametric map restricts to

$$
\left(\left[u_{0}: u_{1}: u_{2}\right],[0: 1]\right) \mapsto\left[0: u_{0}: u_{2}: u_{1}: i u_{2}\right],
$$

a totally real embedding of a real projective plane in the complex projective plane $\mathcal{P}_{2}=\left\{Z_{0}=Z_{2}+i Z_{4}=0\right\}$. Any complex projective line in $\mathbb{C} P^{4}$ meets this part of the image in at most one point, or in a real line or circle.

The final interesting property of the image $\left(Q_{1} \circ s \circ(\delta \times \delta)\right)\left(\mathbb{R} P^{2} \times \mathbb{R} P^{1}\right)$ is that if a complex projective line $\mathcal{L} \subseteq \mathbb{C} P^{4}$ meets the threefold in finitely many points, then they meet in at most two points. This property is obviously a complex projective invariant.

The first step in proving that the property holds is to recall, as in Example 5.1, that the real threefold is contained in a complex projective quadric hypersurface, in 
this case, the variety $\mathcal{H}=\left\{Z_{0}\left(Z_{3}+Z_{4}\right)-Z_{1}\left(Z_{2}+i Z_{4}\right)=0\right\}$. Given any line $\mathcal{L}$, if $\mathcal{L}$ is not contained in $\mathcal{H}$, then $\# \mathcal{L} \cap \mathcal{H} \leq 2$, establishing the claim for such lines. So, it only remains to check those lines $\mathcal{L} \subseteq \mathcal{H}$.

The intersection of $\mathcal{H}$ with the hyperplane $\left\{Z_{0}=0\right\}$ is the union of planes $\mathcal{P}_{1} \cup \mathcal{P}_{2}$. If $\mathcal{L}$ is contained in that union, it must be contained in either $\mathcal{P}_{1}$ or $\mathcal{P}_{2}$ (or be equal to their intersection), and it was already mentioned that such lines have the claimed property. If $\mathcal{L}$ is not contained in the hyperplane $\left\{Z_{0}=0\right\}$, then it meets $\left\{Z_{0}=0\right\}$ in exactly one point, and the only possible exception to the claim is that $\mathcal{L}$ could meet the real threefold at exactly two points in $V_{0}$ and exactly one point of $\mathcal{P}_{1} \cup \mathcal{P}_{2}$.

So, suppose $\mathcal{L}$ is the line incident with two given points in the image: $[1: v$ : $\left.u_{1}+u_{2} v: u_{1} v-u_{2}: u_{2}(1+i v)\right] \in V_{0}$ and $\left[0: r_{0}: r_{2}: r_{1}: i r_{2}\right] \in \mathcal{P}_{2}$, with parametric equation

$\left[z_{0}: z_{1}\right] \mapsto\left[z_{0}: v z_{0}+r_{0} z_{1}:\left(u_{1}+u_{2} v\right) z_{0}+r_{2} z_{1}:\left(u_{1} v-u_{2}\right) z_{0}+r_{1} z_{1}: u_{2}(1+i v) z_{0}+i r_{2} z_{1}\right]$.

Since $\mathcal{L} \subseteq \mathcal{H}$, this parametrization must be identically zero when plugged into the implicit equation for $\mathcal{H}$, which gives

$$
\left(r_{1}-r_{0} u_{1}-i r_{0} u_{2}+i r_{2}\right) z_{0} z_{1}=0
$$

for all $\left[z_{0}: z_{1}\right]$. The conclusion is $\left[r_{0}: r_{1}: r_{2}\right]=\left[1: u_{1}: u_{2}\right]$, but then the two given points are incident with a real line lying on the threefold and contained in $\mathcal{L}$,

$$
\left[t_{0}: t_{1}\right] \mapsto\left[t_{0}: t_{1}: u_{1} t_{0}+u_{2} t_{1}: u_{1} t_{1}-u_{2} t_{0}: u_{2} t_{0}+i u_{2} t_{1}\right],
$$

at $\left[t_{0}: t_{1}\right]=[1: v]$ and $[0: 1]$.

Similarly, suppose $\mathcal{L}$ is the line incident with two given points in the image: $\left[1: v: u_{1}+u_{2} v: u_{1} v-u_{2}: u_{2}(1+i v)\right] \in V_{0}$ and $\left[0: 0: r_{1} s_{0}+r_{2} s_{1}: r_{1} s_{1}-r_{2} s_{0}:\right.$ $\left.r_{2}\left(s_{0}+i s_{1}\right)\right] \in \mathcal{P}_{1}$, so its parametric equation is

$$
\begin{aligned}
{\left[z_{0}: z_{1}\right] \mapsto } & {\left[z_{0}: v z_{0}:\left(u_{1}+u_{2} v\right) z_{0}+\left(r_{1} s_{0}+r_{2} s_{1}\right) z_{1}:\right.} \\
& \left.\left(u_{1} v-u_{2}\right) z_{0}+\left(r_{1} s_{1}-r_{2} s_{0}\right) z_{1}: u_{2}(1+i v) z_{0}+r_{2}\left(s_{0}+i s_{1}\right) z_{1}\right] .
\end{aligned}
$$

Plugging into the implicit equation for $\mathcal{H}$ gives: $\left(s_{1}-s_{0} v\right)\left(r_{1}+i r_{2}\right) z_{0} z_{1}=0$ for all $\left[z_{0}: z_{1}\right]$. The conclusion is $\left[s_{0}: s_{1}\right]=[1: v]$, but then the two given points are incident with a real plane lying on the threefold

$$
\left[t_{0}: t_{1}: t_{2}\right] \mapsto\left[t_{0}: v t_{0}: t_{1}+v t_{2}: v t_{1}-t_{2}:(1+i v) t_{2}\right],
$$

at $\left[t_{0}: t_{1}: t_{2}\right]=\left[1: u_{1}: u_{2}\right]$ and $\left[0: r_{1}: r_{2}\right]$. The line $\mathcal{L}$ meets this real plane along a real projective line incident with the given points. This establishes the claimed property. 
EXAMPLE 5.12. For the family of equivalence classes appearing in Theorem 5.4 in case (6), with $0<t<1$, representative coefficient matrices are of the form:

$$
\mathbf{Q}_{t}=\left[\begin{array}{cccccc}
1 & 0 & 0 & 0 & 0 & 0 \\
0 & 1 & 0 & 0 & 0 & 0 \\
0 & 0 & 1 & 0 & 0 & t^{2} \\
0 & 0 & 0 & 1 & -1 & 0 \\
0 & 0 & 0 & 0 & 1 & i
\end{array}\right]
$$

For each $t$, the induced map $Q_{t} \circ s \circ(\delta \times \delta)$ is given by

$$
\left(\left[u_{0}: u_{1}: u_{2}\right],\left[v_{0}: v_{1}\right]\right) \mapsto\left[u_{0} v_{0}: u_{0} v_{1}: u_{1} v_{0}+t^{2} u_{2} v_{1}: u_{1} v_{1}-u_{2} v_{0}: u_{2} v_{0}+i u_{2} v_{1}\right] .
$$

The singular locus of $Q_{t} \circ s$ is

$$
\Sigma_{4}=\left\{z_{0}=z_{1} w_{0}+i z_{1} w_{1}+i z_{2} w_{0}-t^{2} z_{2} w_{1}=0\right\} .
$$

The image of $\delta \times \delta$ in $\mathbb{C} P^{2} \times \mathbb{C} P^{1}$ is disjoint from $\Sigma_{4}$, so $Q_{t} \circ s \circ(\delta \times \delta)$ is a totally real immersion. The restriction of $Q_{t} \circ s$ to $\Theta=\left\{z_{0}=0\right\}$ is exactly as in Example 4.9 , so we can further conclude that $Q_{t} \circ s \circ(\delta \times \delta)$ is a totally real embedding. For each $r_{3} \in \mathbb{R}$, a complex line in $V_{2}$ of the form $\left\{\left[0: 0: 1: r_{3}: Z_{4}\right]: Z_{4} \in \mathbb{C}\right\}$ meets the image of $Q_{t} \circ s \circ(\delta \times \delta)$ in an ellipse, with eccentricity depending only on $t$. The occurrence of these ellipses is enough to distinguish this image from images with a different value of $t$, or from images in any other r-equivalence class.

In all the rank 2 examples so far, the two-to-one locus of $Q \circ s$ was the set $\left\{z_{1}=0\right\}$, and its intersection with the image of $\delta \times \delta$ was a two-dimensional set of the form $\mathbb{R} P^{1} \times \mathbb{R} P^{1}$, allowing analogies with the examples from Section 4 . In the next two cases, the intersection will be a line, a more generic configuration.

EXAMPLE 5.13. The rank 2 matrix, $\left[\begin{array}{ccc}i & 1 & 0 \\ 0 & 0 & 1\end{array}\right]$ in case (7) of Theorem 5.4, has a representative coefficient matrix

$$
\mathbf{Q}=\left[\begin{array}{cccccc}
0 & 0 & 0 & 0 & 1 & 0 \\
i & 0 & 0 & 0 & 0 & 1 \\
0 & 0 & 1 & 0 & 0 & -1 \\
0 & -4 i & 0 & 0 & 0 & 0 \\
0 & 2 i & 0 & 2 & 0 & 0
\end{array}\right]
$$

The induced map $Q \circ s \circ(\delta \times \delta)$ is given by the formula

$\left(\left[u_{0}: u_{1}: u_{2}\right],\left[v_{0}: v_{1}\right]\right) \mapsto\left[u_{2} v_{0}: i u_{0} v_{0}+u_{2} v_{1}: u_{1} v_{0}-u_{2} v_{1}:-4 i u_{0} v_{1}: 2\left(i u_{0}+u_{1}\right) v_{1}\right]$.

One real line in $\delta_{5}\left(\mathbb{R} P^{5}\right)$ is projected by $Q$ to a point: $Q:\left\{\left[u_{0}: 0: u_{2}: 0: 0:\right.\right.$ $\left.\left.u_{2}\right]\right\} \mapsto[0: 1: 0: 0: 0]$, and this line meets the image of $s \circ(\delta \times \delta)$ exactly once, at $(s \circ(\delta \times \delta))(([0: 0: 1],[1: 0]))=[1: 0: 0: 0: 0: 0]$. 
The two-to-one locus of $Q \circ s$ is $\Theta=\left\{z_{0}-i z_{1}=0\right\}$. The image of $\delta \times \delta$ meets $\Theta$ in the real line $\left\{\left([0: 0: 1],\left[v_{0}+0 i: v_{1}+0 i\right]\right)\right\}$, and the restriction of $Q \circ s$ to this real line is one-to-one: $\left([0: 0: 1],\left[v_{0}: v_{1}\right]\right) \mapsto\left[v_{0}: v_{1}:-v_{1}: 0: 0\right]$, so we can conclude $Q \circ s \circ(\delta \times \delta)$ is one-to-one. The singular locus of $Q \circ s$ is

$$
\Sigma_{4}=\left\{z_{0}-i z_{1}=z_{1} w_{0}+z_{2} w_{1}=0\right\}
$$

which meets the image of $\delta \times \delta$ in exactly one point, ([0:0:1], [1:0]). To check the behavior of $Q \circ s \circ(\delta \times \delta)$ near that point, consider the restriction to the $U_{20} \rightarrow V_{0}$ neighborhoods:

$$
\left(\left[u_{0}: u_{1}: 1\right],[1: v]\right) \mapsto\left[1: i u_{0}+v: u_{1}-v:-4 i u_{0} v: 2\left(i u_{0}+u_{1}\right) v\right] .
$$

Its image in $V_{0}$ is a smoothly embedded graph over the $X_{1}, Y_{1}, X_{2}$ subspace, and the graph is tangent to the origin to this subspace, which contains a complex line, the $Z_{1}$-axis. So, $Q \circ s \circ(\delta \times \delta)$ is an embedding, with exactly one $\mathrm{CR}$ singular point. From a global point of view, this map's image is clearly different from the image of the map from Example 5.10, with the double point and a pair of CR singularities.

The implicit equations for the image in the $Z_{1}, Z_{2}, Z_{3}, Z_{4}$ coordinates of $V_{0}$ are

$$
\left\{Y_{2}=0, Z_{3}=\bar{Z}_{1}^{2}-Z_{1}^{2}, Z_{4}=\left(Z_{1}+\bar{Z}_{1}\right)\left(Z_{1}+X_{2}\right)\right\} .
$$

The image is contained the 5 -subspace $\left\{Y_{2}=X_{3}=2 Y_{4}+Y_{3}=0\right\}$ of $V_{0}$, and each complex line in that subspace is of the form $\left\{\left[1: Z_{1}: r_{2}: i r_{3}: r_{4}-\frac{i}{2} r_{3}\right]: Z_{1} \in \mathbb{C}\right\}$, for some real $r_{2}, r_{3}, r_{4}$. The intersection of such a line with the threefold can be found by solving the system $u_{1}-v=r_{2},-4 i u_{0} v=i r_{3}, 2 i u_{0} v+2 u_{1} v=r_{4}-\frac{i}{2} r_{3}$. If $r_{4} \neq 0$, then there are at most two intersection points. If $r_{4}=0$ and $r_{3} \neq 0$, then there is at most one intersection point, and if $r_{3}=r_{4}=0$, then the line $\left\{\left[1: Z_{1}: r_{2}: 0: 0\right]\right\}$ meets the threefold in a union of a real line,

$$
Q \circ s \circ(\delta \times \delta):\left(\left[u_{0}: r_{2}: 1\right],[1: 0]\right) \mapsto\left[1: i u_{0}: r_{2}: 0: 0\right],
$$

and a (possibly coincident) point,

$$
(Q \circ s \circ(\delta \times \delta))\left(\left([0: 0: 1],\left[1:-r_{2}\right]\right)\right)=\left[1:-r_{2}: r_{2}: 0: 0\right] .
$$

This gives a local way to distinguish this image from Example 5.10; if a curve lies in the intersection of a complex line and the threefold in a neighborhood of the CR singular point, then the curve can only be a real line segment or circular arc, and not something projectively equivalent to an arc of a hyperbola. This CR singularity is non-degenerate, and of type (I), in the sense of [7].

EXAMPLE 5.14. The rank 2 matrix, $\left[\begin{array}{ccc}i & 1 & 0 \\ 0 & i & 1\end{array}\right]$, the generic case (8) of Theorem 
5.4 , has a representative coefficient matrix

$$
\mathbf{Q}=\left[\begin{array}{llllll}
i & 0 & 1 & 0 & 0 & 0 \\
0 & 0 & 1 & i & 0 & 0 \\
0 & 0 & 0 & i & 0 & 1 \\
0 & 1 & 0 & 0 & 0 & 0 \\
0 & 0 & 0 & 0 & 1 & 0
\end{array}\right]
$$

The induced map $Q \circ s \circ(\delta \times \delta)$ is given by the formula

$$
\left(\left[u_{0}: u_{1}: u_{2}\right],\left[v_{0}: v_{1}\right]\right) \mapsto\left[i u_{0} v_{0}+u_{1} v_{0}: u_{1} v_{0}+i u_{1} v_{1}: i u_{1} v_{1}+u_{2} v_{1}: u_{0} v_{1}: u_{2} v_{0}\right] .
$$

One real line in $\delta_{5}\left(\mathbb{R} P^{5}\right)$ is projected by $Q$ to a point: $Q:\left\{\left[u_{0}: 0: u_{2}: u_{0}: 0:\right.\right.$ $\left.\left.u_{2}\right]\right\} \mapsto[1: 1: 1: 0: 0]$, and this line is disjoint from the image of $s$.

The two-to-one locus of $Q \circ s$ is $\Theta=\left\{z_{0}-i z_{1}-z_{2}=0\right\}$. The image of $\delta \times \delta$ meets $\Theta$ in the real line $\left\{\left([1: 0: 1],\left[v_{0}+0 i: v_{1}+0 i\right]\right)\right\}$, and the restriction of $Q \circ s$ to this real line is one-to-one: $\left([1: 0: 1],\left[v_{0}: v_{1}\right]\right) \mapsto\left[i v_{0}: 0: v_{1}: v_{1}: v_{0}\right]$, so we can conclude $Q \circ s \circ(\delta \times \delta)$ is one-to-one. The singular locus of $Q \circ s$ is

$$
\Sigma_{4}=\left\{z_{0}-i z_{1}-z_{2}=z_{0} w_{0}+\left(z_{1}+i z_{0}\right) w_{1}=0\right\},
$$

which is disjoint from the image of $\delta \times \delta$, so $Q \circ s \circ(\delta \times \delta)$ is a totally real embedding.

The restriction to the $U_{20} \rightarrow V_{4}$ neighborhoods is given by

$$
\left(\left[u_{0}: u_{1}: 1\right],[1: v]\right) \mapsto\left[i u_{0}+u_{1}: u_{1}(1+i v):\left(i u_{1}+1\right) v: u_{0} v: 1\right] .
$$

Its image in $V_{4}$ is a smoothly embedded graph of quadratic polynomials over the $Y_{0}$, $X_{1}, X_{2} 3$-subspace, and contained in the real 5-subspace $\left\{X_{0}=X_{1}, Y_{1}=Y_{2}, Y_{3}=0\right\}$. Complex lines in $V_{4}$ not contained in the real 5 -subspace can meet the threefold in a real line or at most two points. The complex lines which are contained in the real subspace are of the form $\left\{\left[Z_{0}: Z_{0}+i r_{1}: Z_{0}+r_{2}+i r_{1}: r_{3}: 1\right]: Z_{0} \in \mathbb{C}\right\}$ for some real $r_{1}, r_{2}, r_{3}$, and each such line meets the threefold in at most three points: there are at most three solutions $\left(u_{0}, u_{1}, v\right)$ of the system of equations $Z_{0}=u_{1}+i u_{0}$, $Z_{0}+i r_{1}=u_{1}(1+i v), Z_{0}+r_{2}+i r_{1}=\left(1+i u_{1}\right) v, r_{3}=u_{0} v$. One complex projective line in $\mathbb{C} P^{4}$ (out of many) that meets the image $(Q \circ s \circ(\delta \times \delta))\left(\mathbb{R} P^{2} \times \mathbb{R} P^{1}\right)$ in exactly three points is given by choosing $\left(r_{1}, r_{2}, r_{3}\right)=(2,1,0)$, to get a parametric equation

$$
\left[z_{0}: z_{1}\right] \mapsto\left[z_{0}: z_{0}+2 i z_{1}: z_{0}+(1+2 i) z_{1}: 0: z_{1}\right]
$$

which meets the image at these three points:

$$
\begin{aligned}
{[1: 1] \mapsto[1: 1+2 i: 2+2 i: 0: 1] } & =(Q \circ s \circ(\delta \times \delta))(([0: 1: 1],[1: 2])), \\
{[-1-2 i: 1] \mapsto[-1-2 i:-1: 0: 0: 1] } & =(Q \circ s \circ(\delta \times \delta))(([-2:-1: 1],[1: 0])), \\
{[-2: 1] \mapsto[-2:-2+2 i:-1+2 i: 0: 1] } & =(Q \circ s \circ(\delta \times \delta))(([0:-2: 1],[1:-1])) .
\end{aligned}
$$


The existence of such a line is enough to distinguish this image from the totally real embedding of Example 5.11. The restriction to the $U_{01} \rightarrow V_{3}$ neighborhoods is similar to the $U_{20} \rightarrow V_{4}$ restriction. To distinguish this image from the totally real embedding from Example 5.12, it will be enough to show that no complex line meets this threefold in an ellipse.

The real threefold is contained in the complex projective cubic hypersurface

$$
\begin{aligned}
0= & Z_{0}\left(Z_{0} Z_{2}-Z_{1} Z_{2}+Z_{1} Z_{3}+Z_{2}^{2}-Z_{2} Z_{3}\right) \\
& -Z_{3} Z_{4}^{2}-i Z_{4}\left(Z_{0} Z_{2}+Z_{0} Z_{3}-Z_{1} Z_{2}+Z_{1} Z_{3}+Z_{2} Z_{3}-Z_{3}^{2}\right) .
\end{aligned}
$$

So, any complex line that meets the threefold in more than three points must also be contained in the complex hypersurface. Having seen only real lines and finite sets as intersections with complex lines in $V_{3} \cup V_{4}$, it remains only to check the complex lines contained in the intersection of the hypersurface with the complex projective plane $\left\{Z_{3}=Z_{4}=0\right\}$. That intersection is $\left\{Z_{3}=Z_{4}=Z_{0} Z_{2}\left(Z_{0}+Z_{2}-Z_{1}\right)=0\right\}$, a union of three lines. The complex line $\left\{Z_{0}=Z_{3}=Z_{4}=0\right\}$ meets the image in a real line,

$$
Q \circ s \circ(\delta \times \delta):\left(\left[0: u_{1}: u_{2}\right],[0: 1]\right) \mapsto\left[0: u_{1}: i u_{1}+u_{2}: 0: 0\right],
$$

and the complex line $\left\{Z_{2}=Z_{3}=Z_{4}=0\right\}$ meets the image in another real line,

$$
Q \circ s \circ(\delta \times \delta):\left(\left[u_{0}: u_{1}: 0\right],[1: 0]\right) \mapsto\left[i u_{0}+u_{1}: u_{1}: 0: 0: 0\right],
$$

which is disjoint from the previous real line. The complex line $\left\{Z_{0}+Z_{2}-Z_{1}=Z_{3}=\right.$ $\left.Z_{4}=0\right\}$ meets the image in exactly two points, $(Q \circ s \circ(\delta \times \delta))(([0: 1: 0],[0: 1]))=$ $[0: 1: 1: 0: 0]$, and $(Q \circ s \circ(\delta \times \delta))(([0: 1: 0],[1: 0]))=[1: 1: 0: 0: 0]$, which are on the above real lines.

The following corollaries of Theorem 5.4 summarize some of the observations from the previous examples. The first one is an analogue of Corollary 4.10.

Corollary 5.15. Given a coefficient matrix $\mathbf{P}_{5 \times 6}$, there exists $B \in P G L(5, \mathbb{C})$ such that one of the two cases holds:

1. the image $(B \circ P \circ s \circ(\delta \times \delta))\left(\mathbb{R} P^{2} \times \mathbb{R} P^{1}\right)$ is contained in a real projective quadratic (Example 5.5) or cubic (Example 5.9) hypersurface in $\delta_{4}\left(\mathbb{R} P^{4}\right)$, or,

2. there exist a real 5-subspace $H$ of the affine neighborhood $V_{0}$, and a real linear coordinate system $\left(x_{1}, x_{2}, x_{3}, x_{4}, x_{5}\right)$ for $H$, such that the intersection of the image $(B \circ P \circ s \circ(\delta \times \delta))\left(\mathbb{R} P^{2} \times \mathbb{R} P^{1}\right)$ and $V_{0}$ is the real affine variety $\left\{x_{4}=x_{1} x_{3}, x_{5}=x_{2} x_{3}\right\} \subseteq H$.

The next corollary strengthens the converse part of Proposition 2.12 in the $\ell=2$, $m=1, n=4$ case. It states that, unlike the cases from Section 4 or Example 2.13, the r-equivalence class of a matrix $\mathbf{P}$ is determined by the image of the induced map $P \circ s \circ(\delta \times \delta)$. 
Corollary 5.16. Given coefficient matrices $\mathbf{P}_{5 \times 6}$ and $\mathbf{Q}_{5 \times 6}$, if there exists $B \in P G L(5, \mathbb{C})$ such that the images of $Q \circ s \circ(\delta \times \delta)$ and $B \circ P \circ s \circ(\delta \times \delta)$ are the same, then $\mathbf{P}$ and $\mathbf{Q}$ are r-equivalent.

\section{REFERENCES}

[1] W. Adkins, J.W. Hoffman, and H.H. Wang. Equations of parametric surfaces with base points via syzygies. J. Symbolic Comput., 39(1):73-101, 2005.

[2] M.A. Akivis and V.V. Goldberg. Differential Geometry of Varieties with Degenerate Gauss Maps. Springer-Verlag, New York, 2004.

[3] F. Aries and R. Senoussi. An implicitization algorithm for rational surfaces with no base points. J. Symbolic Comput., 31(4):357-365, 2001.

[4] E. Bishop. Differentiable manifolds in complex Euclidean space. Duke Math. J., 32(1):1-21, 1965.

[5] A. Coffman. CR singular immersions of complex projective spaces. Beiträge zur Algebra und Geometrie, 43(2):451-477, 2002.

[6] A. Coffman. Real congruence of complex matrix pencils and complex projections of real Veronese varieties. Linear Algebra Appl., 370:41-83, 2003.

[7] A. Coffman. CR singularities of real threefolds in $\mathbb{C}^{4}$. Advances in Geometry, 6(1):109-137, 2006.

[8] A. Coffman and M. Frantz. Möbius transformations and ellipses. The Pi Mu Epsilon Journal, 12(6):339-345, 2007.

[9] D. Cox. Equations of parametric curves and surfaces via syzygies. Symbolic Computation: Solving Equations in Algebra, Geometry, and Engineering, Contemp. Math., 286:1-20, 2001.

[10] E. Elmroth and B. Kågström. The set of 2-by-3 matrix pencils - Kronecker structures and their transitions under perturbations. SIAM J. Matrix Anal. Appl., 17(1):1-34, 1996.

[11] F. Gantmacher. The Theory of Matrices, Vol. II. Chelsea, New York, 1959.

[12] J. Harris. Algebraic Geometry: a First Course. Springer, New York, 1992.

[13] R.A. Horn and C.R. Johnson. Matrix Analysis. Cambridge University Press, Cambridge, 1985.

[14] R.A. Horn and C.R. Johnson. Topics in Matrix Analysis. Cambridge University Press, Cambridge, 1991.

[15] J.G. Semple and L. Roth. Introduction to Algebraic Geometry. Oxford University Press, Oxford, 1985.

[16] W. Waterhouse. Real classification of complex quadrics. Linear Algebra Appl., 48:45-52, 1982. 